The Astrophysical Journal, 681:1470-1483, 2008 July 10

(C) 2008. The American Astronomical Society. All rights reserved. Printed in U.S.A.

\title{
SPITZER IRAC OBSERVATIONS OF WHITE DWARFS. II. MASSIVE PLANETARY AND COLD BROWN DWARF COMPANIONS TO YOUNG AND OLD DEGENERATES
}

\author{
J. FARIhI, ${ }^{1,2}$ E. E. Becklin, ${ }^{1}$ AND B. ZuCKerman ${ }^{1}$ \\ Received 2008 February 23; accepted 2008 March 31
}

\begin{abstract}
This paper presents a sensitive and comprehensive IRAC 3-8 $\mu \mathrm{m}$ photometric survey of white dwarfs for companions in the planetary-mass regime with temperatures cooler than the known $\mathrm{T}$ dwarfs. The search focuses on descendents of intermediate-mass stars with $M \gtrsim 3 M_{\odot}$ whose inner, few hundred AU regions cannot be probed effectively for massive planets and brown dwarfs by any alternative existing method. Furthermore, examination for mid-infrared excess explores an extensive range of orbital semimajor axes, including the intermediate 5-50 AU range, poorly covered and incompletely accessible by other techniques at main-sequence or evolved stars. Three samples of white dwarfs are chosen which together represent relatively young as well as older populations of stars: nine open cluster white dwarfs, 22 high-mass field white dwarfs, and 17 metal-rich field white dwarfs. In particular, these targets include: seven Hyades and four field white dwarfs of similar age, one Pleiades and 19 field white dwarfs of similar age, and van Maanen 2 and 16 similarly metal-rich white dwarfs with ages between 1 and 7 Gyr. No substellar companion candidates were identified at any star. By demanding a $15 \%$ minimum photometric excess at $4.5 \mu \mathrm{m}$ to indicate a companion detection, upper limits in the planetary-mass regime are established at 34 of the sample white dwarfs, 20 of which have limits below $10 M_{\mathrm{J}}$ according to substellar cooling models. Specifically, limits below the minimum mass for deuterium burning are established at all Pleiades and Hyades white dwarfs, as well as similarly young field white dwarfs, half a dozen of which receive limits at or below $5 M_{\mathrm{J}}$. Two IRAC epochs at vMa 2 rule out $T \gtrsim 200 \mathrm{~K}$ proper-motion companions within $1200 \mathrm{AU}$.
\end{abstract}

Subject headings: binaries: general — infrared: stars — planetary systems — stars: evolution stars: low-mass, brown dwarfs — white dwarfs

\section{INTRODUCTION}

The first strong candidate and certain substellar objects identified outside the solar system were all discovered orbiting evolved degenerate stars: the probable brown dwarf companion to the white dwarf GD 165 (Kirkpatrick et al. 1999; Becklin \& Zuckerman 1988) and the planetary system orbiting the pulsar PSR $1257+12$ (Wolszczan \& Frail 1992). Furthermore, the first directly detected, unambiguous substellar and planetary-mass objects were imaged as wide companions orbiting low-luminosity primaries: the brown dwarf secondary to the M dwarf Gl 229 (Nakajima et al. 1995) and the planetary-mass secondary to the young brown dwarf commonly known as 2M1207 (Song et al. 2006; Chauvin et al. 2005). Historically, as well as astrophysically, these properties provide a clear advantage over other types of primaries in the quest to directly detect radiation from bound substellar objects of the lowest mass, such as planets.

Observations indicate that bound substellar objects and planetary system components survive post-main-sequence evolution. First, there now exist roughly one dozen first-ascent giant stars known to harbor substellar and planetary companions (Niedzielski et al. 2007; Reffert et al. 2006; Hatzes et al. 2005, 2006; Sato et al. 2003; Frink et al. 2002). Second, there are at least three white dwarfs which have close, unaltered, and unevolved substellar companions (Burleigh et al. 2006; Maxted et al. 2006; Farihi et al. 2005a). Third, there are at least 10 white dwarfs with infrared excess due to debris disks, which indicate a

\footnotetext{
1 Department of Physics and Astronomy, University of California, 430 Portola Plaza, Los Angeles, CA 90095; jfarihi@astro.ucla.edu, becklin@astro.ucla.edu, ben@astro.ucla.edu.

2 Gemini Observatory, Northern Operations, 670 North A'ohoku Place, Hilo, HI 96720.
}

growing probability of orbiting rocky planetesimals (Farihi et al. 2008; Jura et al. 2007a, 2007b; Kilic \& Redfield 2007; Reach et al. 2005). These cool white dwarfs with warm orbiting dust also display anomalous photospheric metals which are almost certainly accreted from their circumstellar material. The origin of the orbiting material and the dynamical interactions necessary to bring it close enough to the star to be accreted are consistent with remnant planetary systems (Jura 2003; Debes \& Sigurdsson 2002).

The Earth-size radii and consequent low luminosities typical of white dwarfs are clear advantages when searching for light emitted from cold Jupiter-size planets and brown dwarfs. Prior to the launch of Spitzer, generally speaking, only M and L dwarf companions could be detected directly as excess infrared emission from white dwarfs (Tremblay \& Bergeron 2007; Hoard et al. 2007; Burleigh et al. 2006; Farihi et al. 2005a, 2005b, 2006; Farihi 2004; Wachter et al. 2003; Zuckerman \& Becklin 1987; Probst 1983). As a benchmark, a typical $10,000 \mathrm{~K}$ degenerate and an L5 dwarf are about equally luminous at $K$ band (Dahn et al. 2002; Bergeron et al. 1995b), while ground-based observations of white dwarfs at longer wavelengths (where the contrast for cooler companions would improve) are prohibited by overwhelming sky brightness (Glass 1999).

Owing to the capabilities of Spitzer (Werner et al. 2004), a Cycle 1 Infrared Array Camera (IRAC; Fazio et al. 2004) program was undertaken to photometrically search for massive planets and cold substellar companions to relatively young and old white dwarfs, respectively. Specifically, the target sample includes white dwarfs in the Hyades and Pleiades, high-mass field white dwarfs, and metal-rich field white dwarfs. Farihi et al. (2008, hereafter Paper I) describe photometry for all the older (metal-rich) degenerate targets, while this paper presents a synopsis of the IRAC results for the younger (open cluster and high-mass) degenerates, 
TABLE 1

Young Cluster White Dwarf Targets

\begin{tabular}{|c|c|c|c|c|c|}
\hline WD Number & Name & $\begin{array}{c}T_{\text {eff }} \\
(\mathrm{K})\end{array}$ & $\begin{array}{c}V \\
\text { (mag) }\end{array}$ & $\begin{array}{c}M \\
\left(M_{\odot}\right)\end{array}$ & References \\
\hline $0349+247 \ldots$ & EG 25 & 31,700 & 16.64 & 1.09 & $1,2,3$ \\
\hline $0352+096 \ldots \ldots \ldots \ldots \ldots \ldots$ & EG 26 & 14,800 & 14.52 & 0.71 & $1,2,4$ \\
\hline $0406+169 \ldots \ldots \ldots \ldots \ldots \ldots$ & EG 29 & 15,200 & 15.35 & 0.80 & $1,2,4$ \\
\hline $0415+271^{\mathrm{a}} \ldots \ldots \ldots \ldots \ldots$ & EG 265 & 11,500 & 15.00 & 0.55 & $5,6,7$ \\
\hline $0421+162 \ldots \ldots \ldots \ldots \ldots \ldots$ & EG 36 & 19,600 & 14.29 & 0.68 & $1,2,4$ \\
\hline $0425+168 \ldots \ldots \ldots \ldots \ldots \ldots$ & EG 37 & 24,400 & 14.02 & 0.70 & $1,2,4$ \\
\hline $0431+126 \ldots \ldots \ldots \ldots \ldots \ldots$ & EG 39 & 21,300 & 14.24 & 0.65 & $1,2,3$ \\
\hline $0437+138 \ldots \ldots \ldots \ldots \ldots$ & GR 316 & 15,300 & 14.93 & 0.68 & $1,2,7$ \\
\hline $0438+108 \ldots \ldots \ldots \ldots \ldots \ldots$ & EG 42 & 27,400 & 13.86 & 0.75 & $1,2,3$ \\
\hline
\end{tabular}
$\left.{ }^{\mathrm{a}} \mathrm{EG}\right)$.

References.-(1) Claver et al. 2001; (2) Bergeron et al. 1995a; (3) Cheselka et al. 1993; (4) Eggen \& Greenstein 1965; (5) Bergeron et al. 2004; (6) Reid 1993; (7) Upgren et al. 1985.

and upper-mass limits for unresolved companions to all the Cycle 1 targets.

\section{RELATIVELY YOUNG AND OLD DEGENERATE TARGETS}

While highly evolved, white dwarfs are not necessarily old. This fact is exemplified by the nearest and brightest degenerate star in the sky, Sirius B, with a mass of $M \approx 1.00 M_{\odot}$ and a total age of $\tau \approx 240 \mathrm{Myr}$ (Liebert et al. 2005b). For stars which evolved essentially as single objects, there is a correlation between main-sequence progenitor mass and white dwarf mass, derived primarily from studies of open clusters (Dobbie et al. 2006a; Kalirai et al. 2005; Williams et al. 2004; Claver et al. 2001; Weidemann 1987, 1990, 2000). This initial-to-final mass relation yields an estimate of the total age for any particular white dwarf provided its mass and cooling age are accurately known. For white dwarf masses below $0.6 M_{\odot}$, the initial-to-final mass relation is quite steep, and small errors in degenerate mass can lead to large errors in main-sequence lifetime (Burleigh et al. 2002).

\subsection{Hyades and Pleiades Targets}

The Hyades and Pleiades are relatively young and nearby open clusters: the former at $d=46 \mathrm{pc}$ and $\tau=625 \mathrm{Myr}$ (Perryman et al. 1998; Pinsonneault et al. 1998), and the latter at $\tau=125$ Myr and $d=132 \mathrm{pc}$ (Stauffer et al. 1998; Pinsonneault et al. 1998; Soderblom et al. 1998). Classically, the only Pleiades white dwarf is EG 25 (LB 1497), but recently the possibility has been raised that the massive white dwarfs GD 50 and PG 0136+251 may have originated in the same region which gave rise to the cluster (Dobbie et al. 2006b). The latter stars are considered in the following section, and only EG 25 is listed among cluster targets. Table 1 lists all observed open cluster white dwarfs, including the seven classical single Hyades white dwarfs and EG 265 (V411 $\tau$ ), which is either a proper cluster member or part of the Hyades supercluster (Reid 1993; Eggen 1984). Excluded are the Hyades white dwarfs in binary systems: V471 $\tau$ and HZ 9. The open cluster targets come from this study (Spitzer program 3549; PI: E. E. Becklin), with the exception of Hyades targets EG 39 and EG 42, which were extracted from the Spitzer archive (program 2313; PI: M. J. Kuchner).

\subsection{High-Mass Targets}

As with Sirius B and the Pleiades white dwarf, a white dwarf mass near $1.0 M_{\odot}$ implies a short main-sequence lifetime for
TABLE 2

Young Field White Dwarf Targets

\begin{tabular}{|c|c|c|c|c|c|}
\hline WD Number & Name & $\begin{array}{l}T_{\text {eff }} \\
(\mathrm{K})\end{array}$ & $\begin{array}{c}V \\
(\mathrm{mag})\end{array}$ & $\begin{array}{c}M \\
\left(M_{\odot}\right)\end{array}$ & References \\
\hline $0001+433$. & EUVE & 42,400 & 16.8 & 1.37 & $1,2,3$ \\
\hline $0136+251$. & PG & 39,400 & 15.87 & 1.32 & $1,3,4$ \\
\hline $0235-125 \ldots \ldots \ldots \ldots$ & PHL 1400 & 32,400 & 14.98 & 1.03 & $1,2,5$ \\
\hline $0236+498 \ldots \ldots \ldots \ldots \ldots$ & EUVE & 33,800 & 15.8 & 0.94 & 6,7 \\
\hline $0325-857 \mathrm{~A} \ldots \ldots \ldots \ldots . . . . . .$. & LB 9802A & 16,200 & 14.11 & 0.85 & 8,9 \\
\hline $0325-857 \mathrm{~B} \ldots \ldots \ldots \ldots$ & LB 9802B & 33,800 & 14.90 & 1.33 & $1,8,10$ \\
\hline $0346-011 \ldots \ldots \ldots \ldots$ & GD 50 & 43,200 & 14.04 & 1.37 & 1,2 \\
\hline $0440-038 \ldots \ldots \ldots \ldots .$. & EUVE & 65,100 & 16.7 & 1.33 & $1,2,7$ \\
\hline 0518-105 …............ & EUVE & 33,000 & 15.82 & 1.07 & $1,2,3$ \\
\hline $0531-022 \ldots \ldots \ldots \ldots \ldots$ & EUVE & 29,700 & 16.20 & 0.97 & 6,7 \\
\hline $0652-563 \ldots \ldots \ldots \ldots$ & EUVE & 35,200 & 16.6 & 1.18 & 1,2 \\
\hline $0730+487 \ldots \ldots \ldots \ldots \ldots$ & GD 86 & 15,500 & 14.96 & 0.90 & 11 \\
\hline $0821-252 \ldots \ldots \ldots \ldots \ldots$ & EUVE & 43,200 & 16.4 & 1.21 & 12 \\
\hline 0914-195 …............ & EUVE & 56,400 & 17.4 & 1.33 & 1,2 \\
\hline $1022-301 \ldots \ldots \ldots \ldots$ & EUVE & 35,700 & 15.9 & 1.27 & $1,6,7$ \\
\hline $1440+753^{\mathrm{a}} \ldots \ldots \ldots \ldots \ldots$ & EUVE & 35,000 & 15.22 & 1.06 & $3,12,13$ \\
\hline $1529-772 \ldots \ldots \ldots \ldots \ldots$ & EUVE & 51,600 & 16.9 & 1.24 & 2,12 \\
\hline $1543-366 \ldots \ldots \ldots \ldots \ldots$ & EUVE & 45,200 & 15.81 & 1.17 & 6,14 \\
\hline $1609+631 \ldots \ldots \ldots \ldots \ldots$ & PG & 30,400 & 16.68 & 1.05 & 4,6 \\
\hline $1642+413^{\mathrm{b}} \ldots \ldots \ldots \ldots \ldots$ & PG & 26,500 & 16.21 & 0.96 & 4,6 \\
\hline $1658+440 \ldots \ldots \ldots \ldots \ldots$ & PG & 30,500 & 15.02 & 1.41 & 1,4 \\
\hline $1740-706 \ldots \ldots \ldots \ldots$ & EUVE & 46,800 & 16.51 & 1.18 & $1,12,14$ \\
\hline
\end{tabular}

${ }^{\text {a }}$ WD $1440+753$ is a close double degenerate.

b Finley et al. (1997) list $0.96 M_{\odot}(109 \mathrm{pc})$ for $1642+413$, while Liebert et al. (2005a) give $0.79 M_{\odot}(145 \mathrm{pc})$.

ReFERENCES.- (1) Hansen et al. 2006; (2) Vennes et al. 1997; (3) Marsh et al. 1997; (4) Liebert et al. 2005a; (5) Dupuis et al. 2002; (6) Finley et al. 1997; (7) McCook \& Sion 2006; (8) Barstow et al. 1995; (9) Ferrario et al. 1997; (10) Vennes et al. 2003; (11) Bergeron et al. 1992; (12) Vennes 1999; (13) Vennes et al. 1999; (14) Vennes et al. 1996.

unadulterated single star evolution, regardless of association. More specifically, were these young white dwarfs identical in mass (depending on the reference, their masses differ by no more than 10\%; Liebert et al. 2005b; Claver et al. 2001), their total age difference can be estimated from the difference in their effective temperatures: $T_{\text {eff }}=25,200 \mathrm{~K}$ for Sirius B, and $T_{\text {eff }}=31,700 \mathrm{~K}$ for EG 25. For these temperatures, $\log g=8.6$ (very nearly $1.0 M_{\odot}$ ) hydrogen atmosphere models predict cooling ages of 130 and $60 \mathrm{Myr}$, respectively, which by itself would account for $60 \%$ of their total age difference (Barstow et al. 2005; Claver et al. 2001; Bergeron et al. 1995a, 1995b, 1995c). In reality the total difference in their ages is due both to differential cooling and unequal main-sequence progenitor lifetimes.

Any hot, high-mass white dwarf which evolved as a single star will be similarly young, or even younger for higher temperatures or masses. In this paper, it is assumed that all high-mass white dwarfs are descended from single main-sequence star progenitors of intermediate mass, but this may not be the case. There appears to be indirect evidence in favor of, as well as against, the existence of high-mass white dwarfs resulting from mergers, but no direct evidence exists (Hansen et al. 2006; Ferrario et al. 2005; Liebert et al. 2005a). Ten hot and massive white dwarf targets come from this study (Spitzer program 3549; PI: E. E. Becklin), and one dozen similar targets were extracted from the Spitzer archive (program 3309; PI: B. Hansen). Table 2 lists the 22 hot field white dwarfs with masses $M \geq 0.9 M_{\odot}$ selected for study.

\subsection{Older Targets}

Included in the sample are metal-rich white dwarfs from Paper I (Spitzer program 3548; PI: B. Zuckerman) with the addition of 
TABLE 3

Metal-Rich Field White Dwarf Targets

\begin{tabular}{|c|c|c|c|c|c|}
\hline WD Number & Name & $\begin{array}{c}T_{\text {eff }} \\
(\mathrm{K})\end{array}$ & $\begin{array}{c}V \\
\text { (mag) }\end{array}$ & $\begin{array}{c}M \\
\left(M_{\odot}\right)\end{array}$ & References \\
\hline $0032-175 \ldots \ldots$ & G266-135 & 9240 & 14.94 & 0.60 & 1,2 \\
\hline 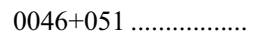 & vMa 2 & 6770 & 12.39 & 0.83 & 3 \\
\hline $0235+064 \ldots \ldots \ldots \ldots \ldots \ldots$ & PG & 15000 & 15.5 & 0.61 & 4 \\
\hline $0322-019 \ldots \ldots \ldots \ldots \ldots$ & G77-50 & 5220 & 16.12 & 0.61 & 5,6 \\
\hline $0846+346 \ldots \ldots \ldots \ldots \ldots$ & GD 96 & 7370 & 15.71 & 0.59 & 1,7 \\
\hline $1102-183 \ldots \ldots \ldots \ldots \ldots$ & $\mathrm{EC}$ & 8060 & 15.99 & 0.60 & 1,7 \\
\hline 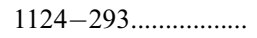 & $\mathrm{EC}$ & 9680 & 15.02 & 0.63 & 5,8 \\
\hline $1204-136 \ldots \ldots \ldots \ldots \ldots$ & $\mathrm{EC}$ & 11500 & 15.67 & 0.60 & 1,9 \\
\hline $1208+576 \ldots \ldots \ldots \ldots \ldots$ & G197-47 & 5880 & 15.78 & 0.56 & 3 \\
\hline $1344+106 \ldots \ldots \ldots \ldots \ldots$ & G63-54 & 7110 & 15.12 & 0.65 & 3 \\
\hline $1407+425 \ldots \ldots \ldots \ldots \ldots \ldots$ & PG & 10010 & 15.03 & 0.73 & 10 \\
\hline $1455+298 \ldots \ldots \ldots \ldots \ldots . . . .$. & G167-8 & 7390 & 15.60 & 0.58 & 3,10 \\
\hline $1632+177 \ldots \ldots \ldots \ldots \ldots$ & PG & 10100 & 13.05 & 0.58 & 10 \\
\hline 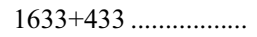 & G180-63 & 6690 & 14.84 & 0.72 & 3,10 \\
\hline $1826-045 \ldots \ldots \ldots \ldots \ldots$ & G21-16 & 9480 & 14.58 & 0.57 & 3 \\
\hline 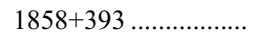 & G205-52 & 9470 & 15.63 & 0.60 & 1,7 \\
\hline $2326+049 \ldots \ldots \ldots \ldots \ldots$ & G29-38 & 11600 & 13.04 & 0.69 & 7,11 \\
\hline
\end{tabular}

NotE.-For those stars with no spectroscopic or trigonometric mass-radius estimate, $\log g=8.0$ was assumed.

REFERENCES.- (1) Zuckerman et al. 2003; (2) Mermilliod 1986; (3) Bergeron et al. 2001; (4) This work; (5) Bergeron et al. 1997; (6) Smart et al. 2003; (7) McCook \& Sion 2006; (8) Koester et al. 2001; (9) Salim \& Gould 2003; (10) Liebert et al. 2005a; (11) Bergeron et al. 1995d.

vMa 2, which was extracted from the Spitzer archive ( program 33; PI: G. G. Fazio). These targets are relatively old, with total ages between 1 and 7 Gyr, and are listed in Table 3 for completeness, although their IRAC fluxes are previously published in Paper I with the exception of vMa 2.

\section{OBSERVATIONS AND DATA}

For white dwarf targets in programs 3548 and 3549, the details of the IRAC observing strategy, data reduction, and analysis are described in full detail in Paper I. In these programs a total exposure time of $600 \mathrm{~s}$ was utilized for each target in all bandpasses. For white dwarf targets extracted from the Spitzer archive, the exposure times were shorter and occasionally in only two bandpasses (see Mullally et al. 2007; Hansen et al. 2006). Fortunately, all targets were unambiguously detected at $4.5 \mu \mathrm{m}$, the wavelength which places the best constraints on spatially unresolved cold substellar and massive planetary companions, according to models for the appropriate range of ages and masses (Burrows et al. 2003; Baraffe et al. 2003).

\subsection{Photometry and Upper Limits}

Paper I contains a detailed discussion regarding the consistency of measured IRAC fluxes of white dwarfs compared to model predictions and concludes the photometric accuracy is well described as 5\%. Also described there is a conservative approach to estimating the signal-to-noise ratio of IRAC detections in the presence of possible confusion and spatially varying background. In Paper I, all targets were detected at all wavelengths, which is not the case here.

To create upper limits for nondetections, aperture photometry was performed as described in Paper I at the nominal location of the white dwarf, derived from one or more IRAC channels in which the source was positively detected. Utilizing the smallest radius ( $r=2$ pixels) for which there are published aperture corrections, the flux in this aperture was compared to the per pixel sky noise multiplied by the area of the aperture, and the larger of these values was taken to be the upper limit, after an appropriate aperture correction. In nearly all cases, the larger value was given by the additive noise in the aperture, but there were a few cases in which there was flux measured above this level. In these cases, it appeared possible or likely that the measured flux originated from background sources, as evidenced by pixel shifts in the centroid of the source flux compared to the other IRAC channels, or sources which were apparently extended.

It is noteworthy that the photometric errors used here, as well as the derived upper limits for nondetections, are somewhat larger than those published in Hansen et al. (2006) and Mullally et al. (2007) for the same observations. In some cases where white dwarf flux is reported by those authors, Table 4 indicates only an upper limit for the reasons stated at the end of the previous paragraph. IRAC fluxes and upper limits for 32 of 48 studied stars (excepting those previously published in Paper I) are listed in Table 4 and plotted in Figures 1-8.

\section{ANALYSIS \\ 4.1. Total Ages}

To infer companion-mass limits using substellar cooling models, each white dwarf target requires an assessment of its total age since formation as a main-sequence star. Substellar companions which form in a binary will be truly coeval, while massive planets are thought to form within $10 \mathrm{Myr}$ of their main-sequence hosts. For the open cluster white dwarfs, their total age is the cluster age, while for field white dwarfs the following methods are used to estimate their ages.

First, any field white dwarf with a mass and effective temperature similar to or higher than the Pleiades white dwarf EG 25 is assumed to be of similar age, roughly $0.1 \mathrm{Gyr}$. There are sufficient uncertainties in both the masses and temperatures of these stars, as evidenced by the $10 \%-20 \%$ variation among parameters cited in the literature for the same objects (see Table 2 for a list of references), which translates into errors in cooling ages of order 10-20 Myr. Fortunately, this type of error should be offset when assessing a total age, because higher mass white dwarfs cool more slowly (smaller surface area) yet have shorter inferred mainsequence lifetimes than their less massive counterparts. For the high-mass field stars considered here, the modest errors in cooling age and inferred main-sequence lifetime, which result from uncertainties in white dwarf parameters, are comparable in magnitude and therefore tend to cancel out. Given these uncertainties for the hot and massive field white dwarfs, it seems prudent to assign a $20 \%$ uncertainty in their total ages, or $\tau=0.125 \pm 0.025$ Gyr.

Second, all cooler and less massive (i.e., older) field degenerates have their total ages assessed following the procedure employed originally by Burleigh et al. (2002) and more recently by Debes et al. (2007). This latter method utilizes the initial-to-final mass to relation to obtain a main-sequence mass from the current, known white dwarf mass. A main-sequence lifetime is then assigned based on the inferred main-sequence mass, and this is added to the white dwarf cooling lifetime to obtain an approximate total age,

$$
\tau=t_{\mathrm{ms}}+t_{\mathrm{wd}}
$$

Cooling ages come from models of P. Bergeron (2002, private communication; Bergeron et al. 1995b, 1995c), while mainsequence lifetimes were calculated using the formulae of Hurley et al. (2000). Tables 5 and 6 list the relevant ages for all targets together with upper-mass limits for substellar companions determined as described below. It should be stated that this general 
TABLE 4

IRAC FluXes For White DWARF TARgets

\begin{tabular}{|c|c|c|c|c|c|}
\hline WD Number & $\begin{array}{c}F_{3.6 \mu \mathrm{m}} \\
(\mu \mathrm{Jy})\end{array}$ & $\begin{array}{c}F_{4.5 \mu \mathrm{m}} \\
(\mu \mathrm{Jy})\end{array}$ & $\begin{array}{c}F_{5.7 \mu \mathrm{m}} \\
(\mu \mathrm{Jy})\end{array}$ & $\begin{array}{c}F_{7.9 \mu \mathrm{m}} \\
(\mu \mathrm{Jy})\end{array}$ & Pipeline \\
\hline $0001+433 \ldots \ldots \ldots \ldots \ldots$ & $18 \pm 6$ & $13 \pm 7$ & $30^{\mathrm{a}}$ & $29^{\mathrm{a}}$ & 14.0 \\
\hline $0046+051 \ldots \ldots \ldots \ldots \ldots$ & $8040 \pm 400$ & $5360 \pm 270$ & $3680 \pm 190$ & $2080 \pm 110$ & 14.0 \\
\hline $0136+251 \ldots \ldots \ldots \ldots \ldots$ & $46 \pm 6$ & $26 \pm 7$ & $34^{\mathrm{a}}$ & $35^{\mathrm{a}}$ & 14.0 \\
\hline $0235-125 \ldots \ldots \ldots \ldots \ldots$ & $111 \pm 8$ & $63 \pm 7$ & $36^{\mathrm{a}}$ & $35^{\mathrm{a}}$ & 14.0 \\
\hline $0236+498 \ldots \ldots \ldots \ldots \ldots$ & $107 \pm 11$ & $72 \pm 9$ & $20^{\mathrm{a}}$ & $27^{\mathrm{a}}$ & 11.0 \\
\hline $0325-857 \mathrm{~A} \ldots \ldots \ldots \ldots$ & $396 \pm 20$ & $238 \pm 14$ & $139 \pm 27$ & $87 \pm 25$ & 14.0 \\
\hline $0325-857 \mathrm{~B} \ldots \ldots \ldots \ldots$ & $149 \pm 9$ & $92 \pm 8$ & $53 \pm 28$ & $33^{\mathrm{a}}$ & 14.0 \\
\hline $0346-011 \ldots \ldots \ldots \ldots \ldots$ & $263 \pm 14$ & $164 \pm 11$ & $145 \pm 34$ & $47^{\mathrm{a}}$ & 14.0 \\
\hline $0349+247 \ldots \ldots \ldots \ldots \ldots$ & $27 \pm 3$ & $15 \pm 4$ & $22^{\mathrm{a}}$ & $39^{\mathrm{a}}$ & 11.4 \\
\hline $0352+096 \ldots \ldots \ldots \ldots \ldots \ldots$ & $295 \pm 15$ & $168 \pm 9$ & $99 \pm 21$ & $44 \pm 28$ & 11.4 \\
\hline $0406+169 \ldots \ldots \ldots \ldots \ldots$ & $134 \pm 7$ & $69 \pm 5$ & $55 \pm 20$ & $61 \pm 34$ & 11.4 \\
\hline $0415+271 \ldots \ldots \ldots \ldots \ldots$ & $245 \pm 13$ & $148 \pm 8$ & $100 \pm 19$ & $68 \pm 23$ & 11.4 \\
\hline $0421+162 \ldots \ldots \ldots \ldots \ldots \ldots$ & $275 \pm 14$ & $163 \pm 9$ & $121 \pm 20$ & $58 \pm 23$ & 11.4 \\
\hline $0425+168 \ldots \ldots \ldots \ldots \ldots$ & $313 \pm 16$ & $185 \pm 10$ & $133 \pm 21$ & $101 \pm 27$ & 11.4 \\
\hline $0431+126 \ldots \ldots \ldots \ldots \ldots \ldots$ & $\ldots$ & $179 \pm 10$ & $\ldots$ & $51^{\mathrm{a}}$ & 14.0 \\
\hline 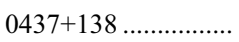 & $186 \pm 10$ & $107 \pm 6$ & $62 \pm 17$ & $42^{\mathrm{a}}$ & 11.4 \\
\hline $0438+108 \ldots \ldots \ldots \ldots \ldots$ & $\ldots$ & $211 \pm 12$ & $\ldots$ & $76 \pm 38$ & 14.0 \\
\hline $0440-038 \ldots \ldots \ldots \ldots \ldots$ & $20 \pm 5$ & $19 \pm 6$ & $23^{\mathrm{a}}$ & $21^{\mathrm{a}}$ & 14.0 \\
\hline $0518-105 \ldots \ldots \ldots \ldots \ldots$ & $51 \pm 5$ & $33 \pm 7$ & $32^{\mathrm{a}}$ & $31^{\mathrm{a}}$ & 14.0 \\
\hline $0531-022 \ldots \ldots \ldots \ldots . . . .$. & $47 \pm 4$ & $26 \pm 5$ & $20^{\mathrm{a}}$ & $37^{\mathrm{a}}$ & 11.4 \\
\hline $0652-563 \ldots \ldots \ldots \ldots \ldots$ & $16 \pm 11$ & $18 \pm 9$ & $32^{\mathrm{a}}$ & $30^{\mathrm{a}}$ & 14.0 \\
\hline $0730+487 \ldots \ldots \ldots \ldots \ldots$ & $218 \pm 11$ & $127 \pm 7$ & $98 \pm 22$ & $66 \pm 24$ & 10.5 \\
\hline $0821-252 \ldots \ldots \ldots \ldots \ldots$ & $26 \pm 10$ & $13 \pm 7$ & $21^{\mathrm{a}}$ & $20^{\mathrm{a}}$ & 11.0 \\
\hline $0914-195$................. & $13 \pm 5$ & $9 \pm 6$ & $33^{\mathrm{a}}$ & $32^{\mathrm{a}}$ & 14.0 \\
\hline $1022-301 \ldots \ldots \ldots \ldots . .$. & $33 \pm 6$ & $22 \pm 7$ & $30^{\mathrm{a}}$ & $31^{\mathrm{a}}$ & 14.0 \\
\hline $1440+753 \ldots \ldots \ldots \ldots \ldots$ & $88 \pm 6$ & $58 \pm 5$ & $27 \pm 16$ & $16^{\mathrm{a}}$ & 11.0 \\
\hline $1529-772 \ldots \ldots \ldots \ldots \ldots$ & $22 \pm 5$ & $12 \pm 4$ & $20^{\mathrm{a}}$ & $18^{\mathrm{a}}$ & 12.4 \\
\hline $1543-366 \ldots \ldots \ldots \ldots$ & $51 \pm 5$ & $31 \pm 5$ & $23^{\mathrm{a}}$ & $23^{a}$ & 11.4 \\
\hline $1609+631 \ldots \ldots \ldots \ldots \ldots$ & $24 \pm 3$ & $13 \pm 3$ & $15^{\mathrm{a}}$ & $22^{\mathrm{a}}$ & 11.0 \\
\hline $1642+413 \ldots \ldots \ldots \ldots \ldots$ & $38 \pm 3$ & $21 \pm 4$ & $16^{\mathrm{a}}$ & $17^{\mathrm{a}}$ & 11.4 \\
\hline $1658+440 \ldots \ldots \ldots \ldots \ldots$ & $131 \pm 8$ & $81 \pm 7$ & $56 \pm 27$ & $28^{\mathrm{a}}$ & 14.0 \\
\hline $1740-706 \ldots \ldots \ldots \ldots \ldots$ & $25 \pm 4$ & $14 \pm 4$ & $16^{\mathrm{a}}$ & $19^{\mathrm{a}}$ & 14.0 \\
\hline
\end{tabular}

NotE.-Photometric errors and upper limits are described in $\S 3.1$.

a Upper limit.
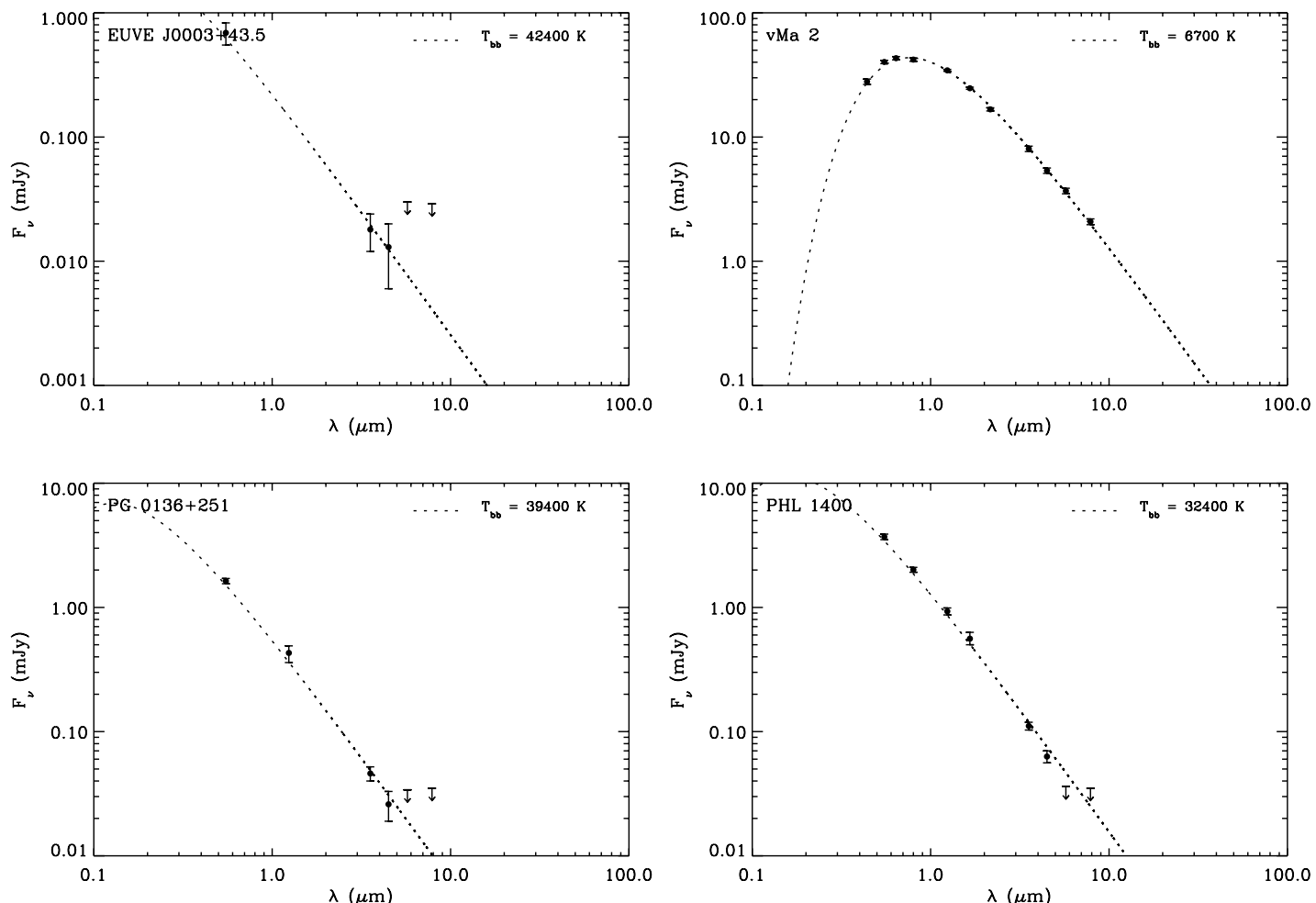

FIG. 1.-Spectral energy distribution of EUVE J0003+43.5, vMa 2, PG 0136+251, and PHL 1400. Downward-pointing arrows represent upper limits ( 3.1$)$. 

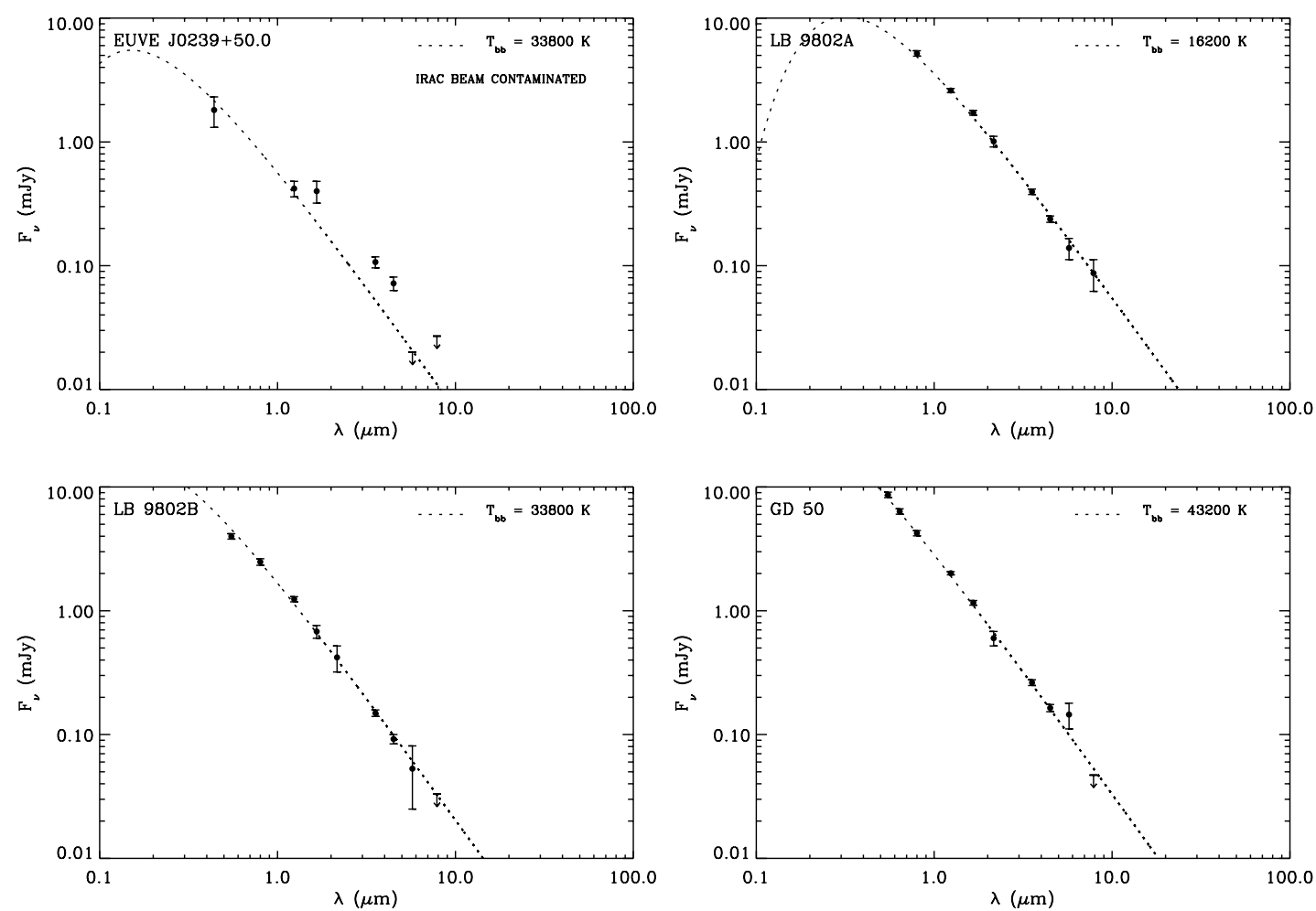

FIG. 2.- Same as Fig. 1, but for EUVE J0239+50.0, LB 9802A, LB 9802B, and GD 50.

method is the best available to estimate the total age of white dwarfs not belonging to open clusters or multiple systems from which another age constraint might be gleaned. However, there are several sources of uncertainty in the estimation of total ages, including but not limited to: the slope of the initial-to-final mass
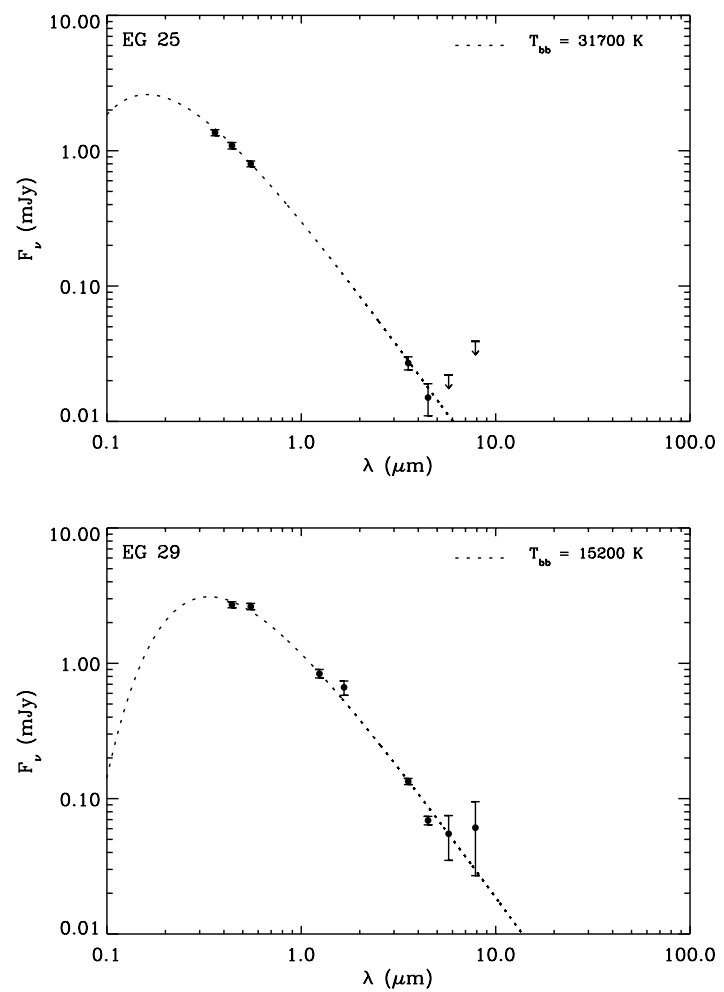

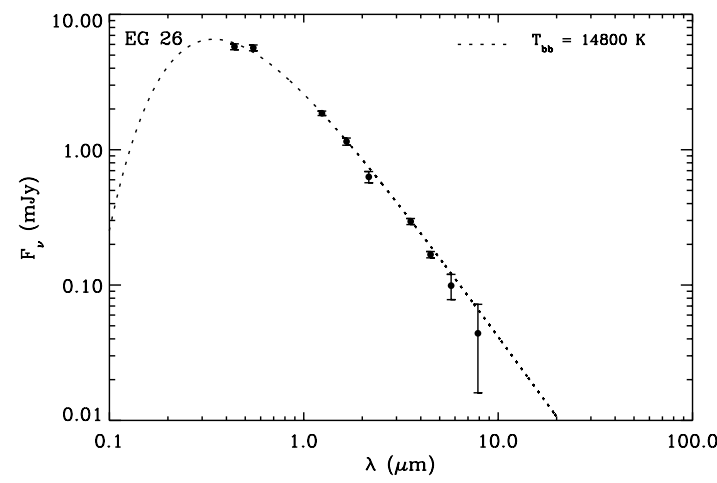

relation, assumed main-sequence lifetimes, and white dwarf model uncertainties; specifically, spectroscopic parameter fits and cooling ages (Kalirai et al. 2008; Dobbie et al. 2006a; Ferrario et al. 2005). Owing to these facts, the uncertainty in the total ages of older field white dwarfs is taken to be $25 \%$.

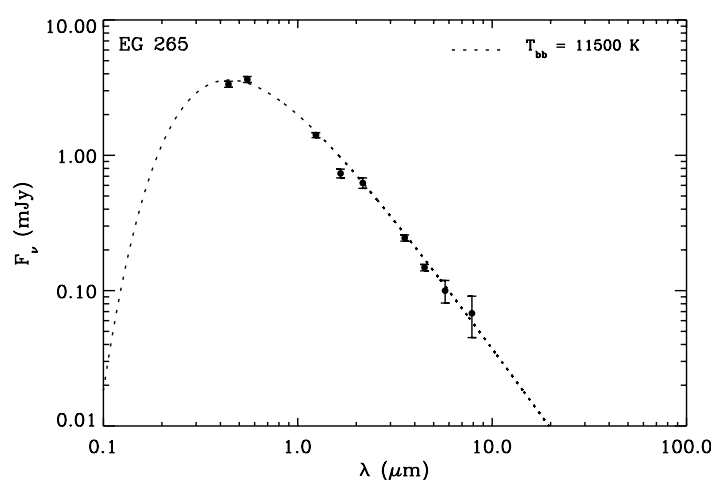

FIG. 3.-Same as Fig. 1, but for EG 25, EG 26, EG 29, and EG 265. 

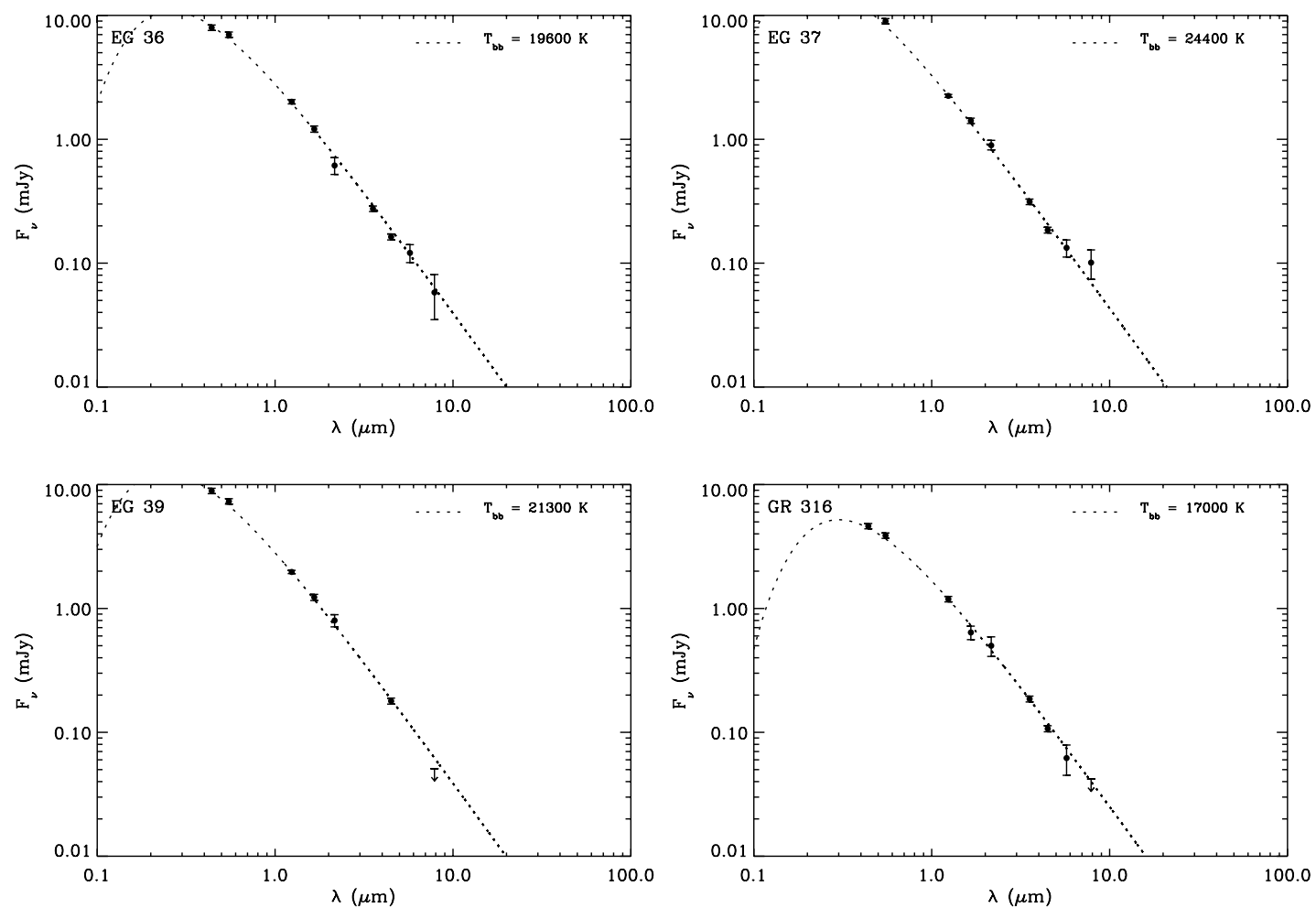

FIG. 4.-Same as Fig. 1, but for EG 35, EG 37, EG 39, and GR 316.

\subsection{Unresolved Companion-Mass Limits from $4.5 \mu \mathrm{m}$ Fluxes}

All white dwarfs in Tables 1-3 have measured IRAC fluxes at $4.5 \mu \mathrm{m}$, where cold $\left(T_{\mathrm{eff}}<1000 \mathrm{~K}\right)$ substellar objects are predicted to be brightest (Baraffe et al. 2003; Burrows et al. 2003). This bandpass is best for placing limits on any spatially unresolved
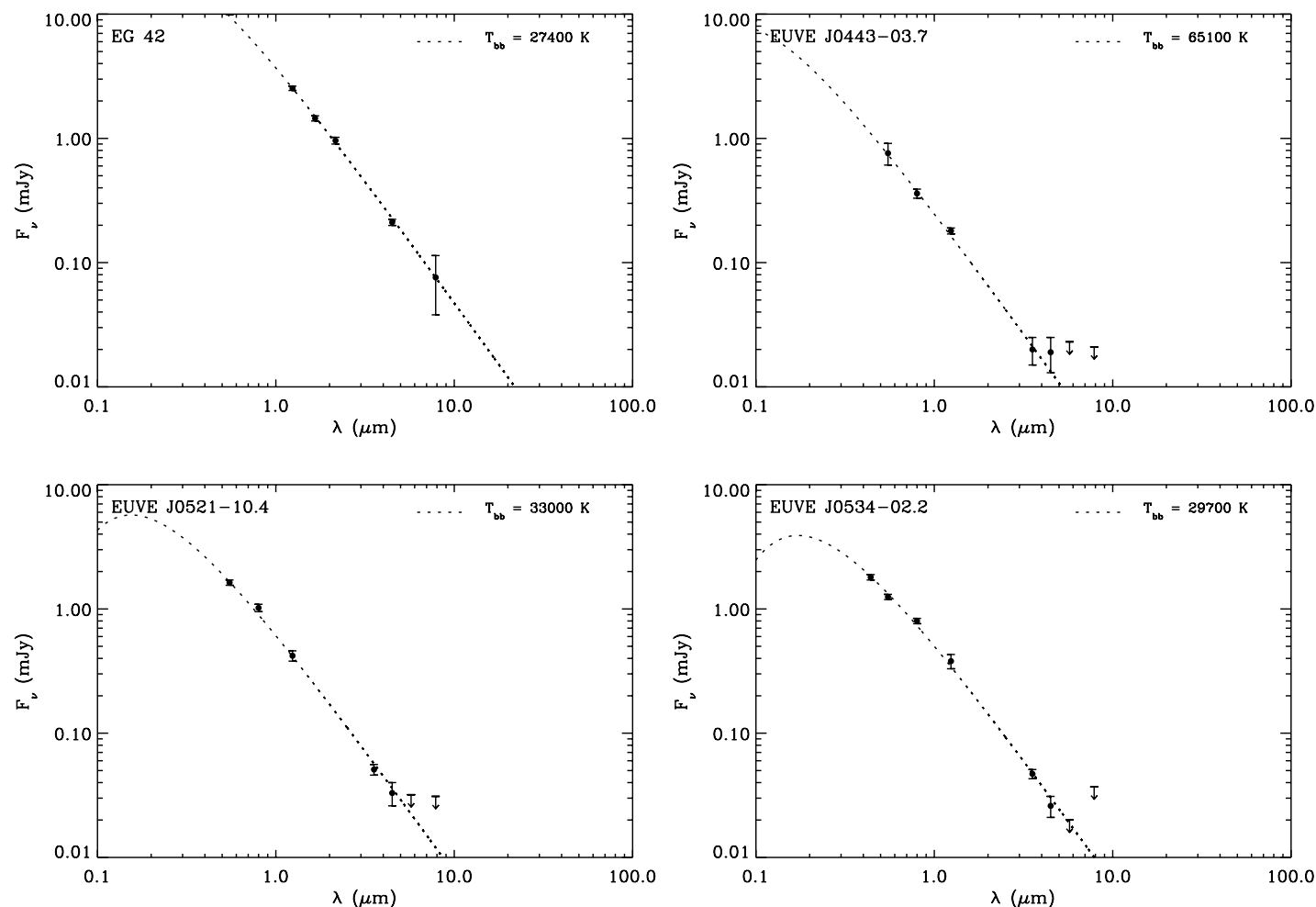

substellar companions. The flux errors in Table 4 are $1 \sigma$ values, but for reasons discussed in Paper I, and to be conservative, an unambiguous detection of excess at this wavelength is defined here as $3 \sigma$ above the expected white dwarfflux. By demanding this level of excess, blackbody models will suffice to predict the expected $4.5 \mu \mathrm{m}$ photospheric flux of the white dwarf targets

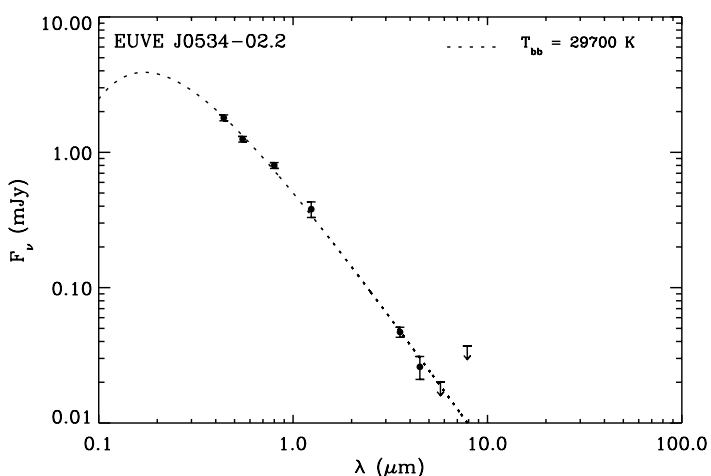

FIG. 5.-Same as Fig. 1, but for EG 42, EUVE J0443-03.7, EUVE J0521- 10.4, and EUVE J0534-02.2. 

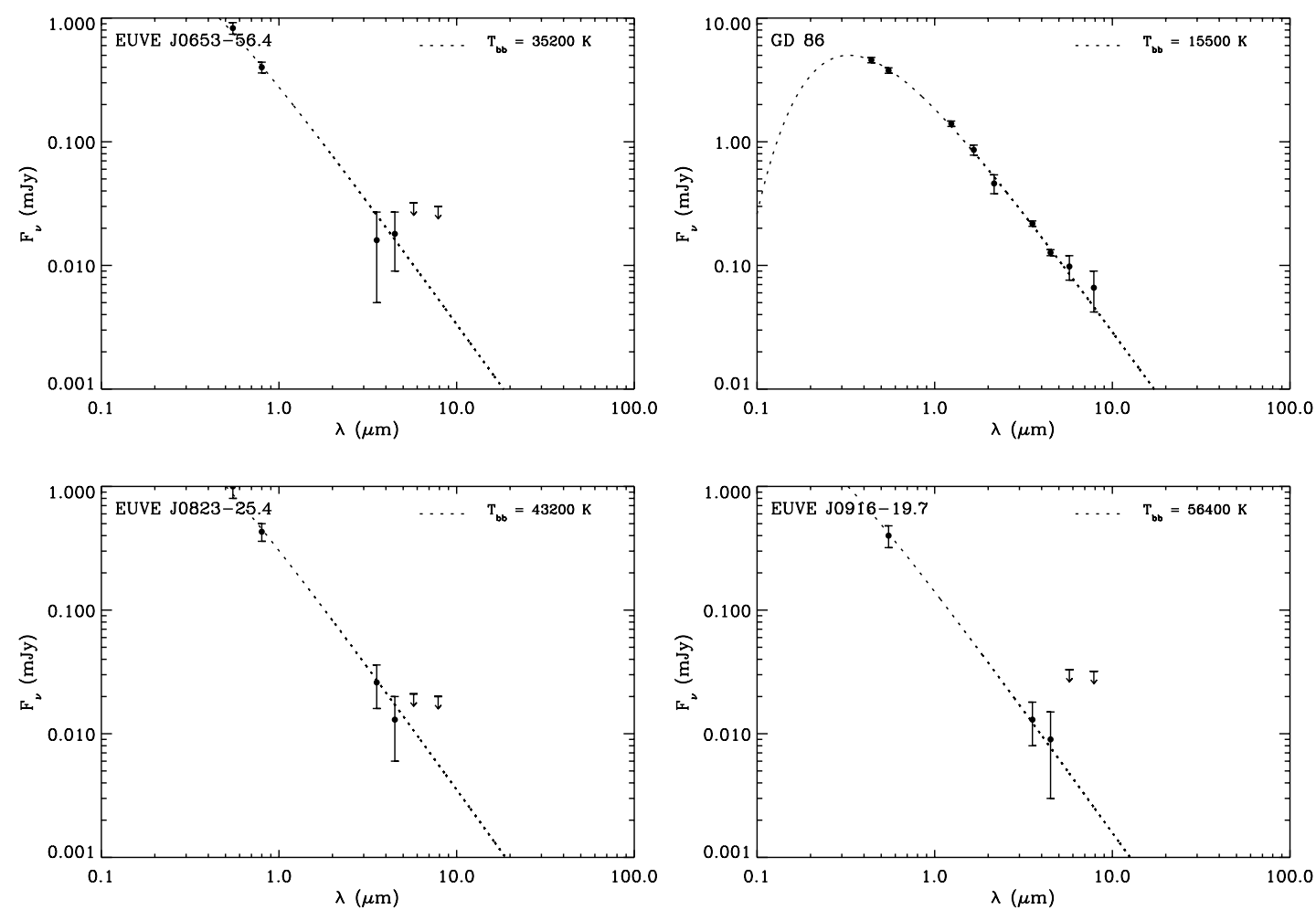

FIG. 6.- Same as Fig. 1, but for EUVE J0653-56.4, GD 86, EUVE J0823-25.4, and EUVE J0916-19.7.

(Paper I). The absolute magnitude corresponding to the minimally reliable excess flux from each white dwarf target is then given by

$M_{4.5 \mu \mathrm{m}}=-2.5 \log \left[\frac{3 \sigma}{F_{0}}\left(\frac{d}{10}\right)^{2}\right]$,
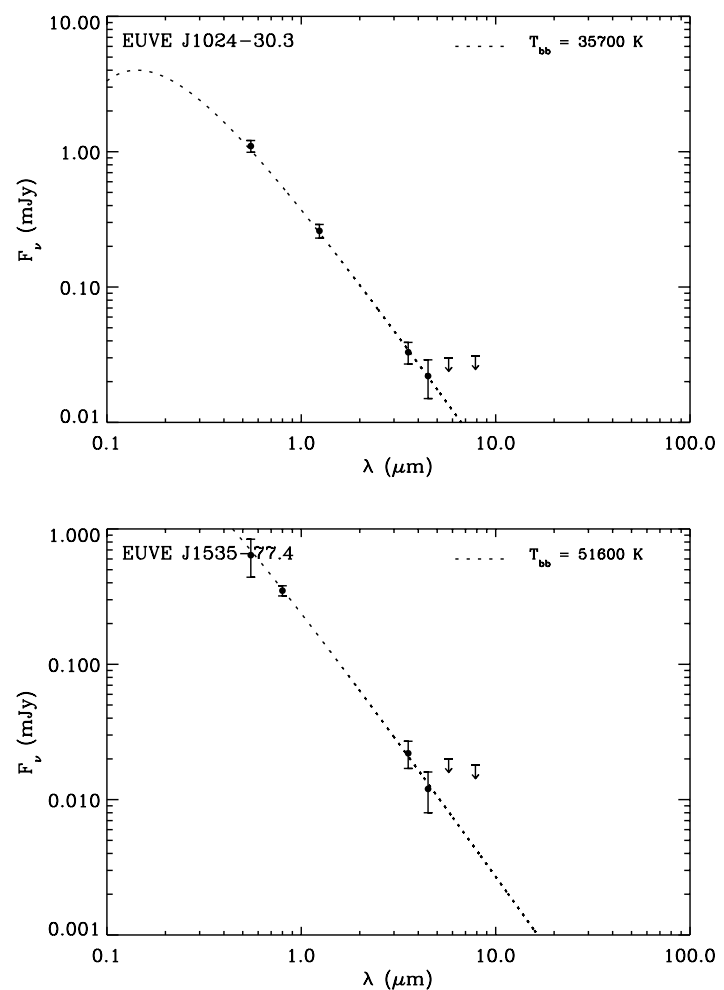

where $\sigma$ is the total flux error in janskys at $4.5 \mu \mathrm{m}$ from Table $4, d$ is the distance to the white dwarf in parsecs, and $F_{0}=179.7 \mathrm{Jy}$ (Spitzer Science Center 2006a). Substellar cooling models updated to include fluxes in the IRAC bandpasses were used to transform the expected flux into a mass for a given age (I. Baraffe 2007, private communication; Baraffe et al. 2003). Some representative,
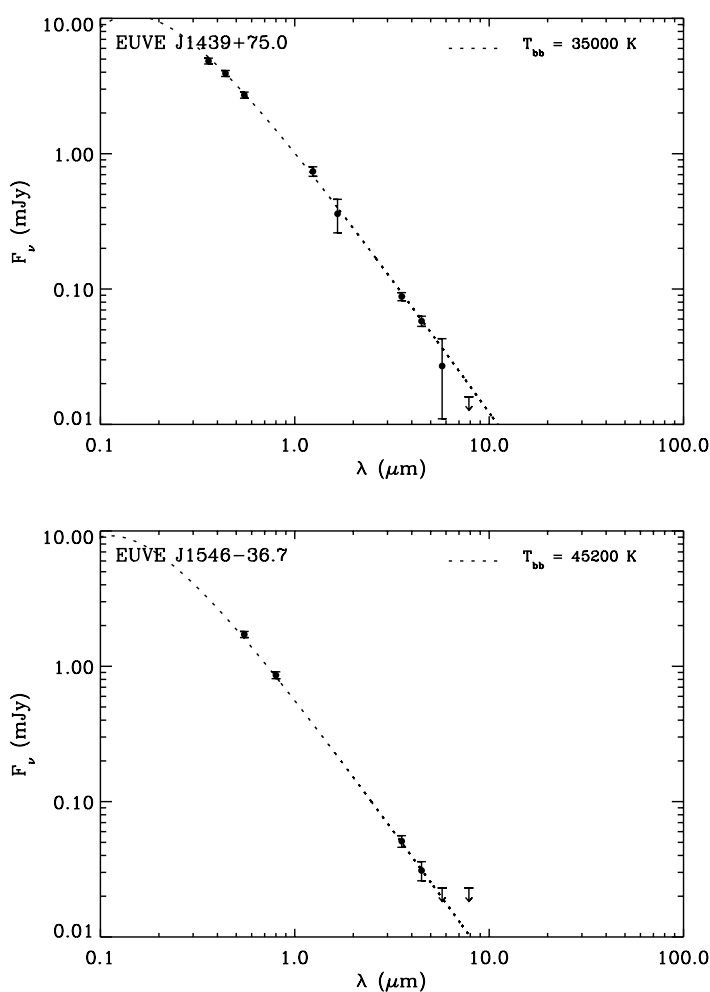

FIG. 7.-Same as Fig. 1, but for EUVE J1024-30.3, EUVE J1439+75.0, EUVE J1535-77.4, and EUVE J1546-36.7. 

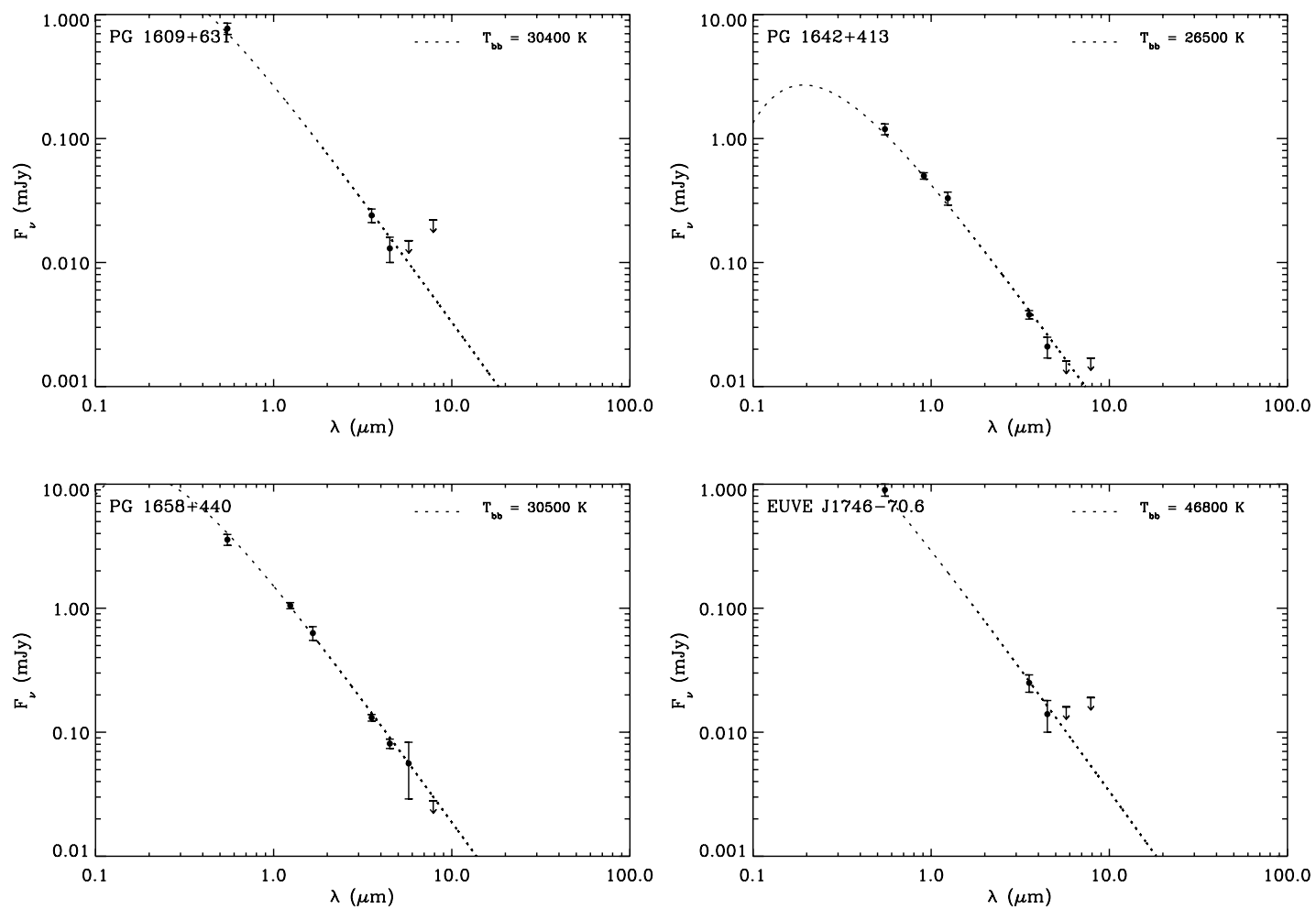

FIG. 8. - Same as Fig. 1, but for PG 1609+631, PG 1642+413, PG 1658+440, and EUVE J1746-70.6.

model-predicted values for $M_{4.5} \mu \mathrm{m}$ at the ages of interest are given in Table 7. It is noteworthy that this analysis rules out unresolved sub-T dwarf companions at 25 white dwarfs in Table 5; the known $\mathrm{T}$ dwarf sequence ends near $M_{4.5} \mu \mathrm{m}=13.5 \mathrm{mag}$ and T8 (Patten et al. 2006), and limits at these stars reach $13.6 \mathrm{mag} \leq M_{4.5 \mu \mathrm{m}} \leq$ $15.2 \mathrm{mag}$ (typically $M_{4.5 \mu \mathrm{m}}=14.3 \pm 0.5 \mathrm{mag}$ ).

In addition, any spatially resolved point sources detected within several arcseconds of the white dwarf were photometrically examined for the possibility of companionship via their IRAC colors and any available ground-based photometric or astrometric data. Owing to the higher sensitivity of the two short IRAC wavelengths, some resolved substellar objects may not be detected at the two long IRAC wavelengths, resulting in a potential ambiguity for some visual companions. Generally speaking, no candidate companions were identified in this manner, but a few possibilities are discussed in $\S 5.7$.

\subsection{Resolved Companion-Mass Limits from $7.9 \mu \mathrm{m}$ Fluxes}

In order to detect $\mathrm{T}$ and sub-T dwarfs as spatially resolved companions, and to differentiate such objects from background pointlike sources, they must be reliably detected at all four IRAC wavelengths. The two long-wavelength IRAC channels in particular, together with the two short-wavelength channels, provide unique information which should eliminate the color degeneracy between cool brown dwarfs and red extragalactic (pointlike) sources in the near-infrared and short-wavelength IRAC channels alone (Patten et al. 2006). This fact appreciably limits any widefield IRAC search for T dwarf companions due to the lower sensitivity of the long-wavelength IRAC channels (Spitzer Science Center 2006b), as detailed below. There are seven white dwarfs from Table 3 which met the necessary criteria for such a search: (1) IRAC imaging of their surrounding fields in all four channels and (2) a distance within approximately $20 \mathrm{pc}$. The entire known $\mathrm{T}$ dwarf sequence (down to T8) should be detected at all four wavelengths at these distances (Patten et al. 2006).
Figure 9 shows the number of detected sources in each IRAC channel, as a function of magnitude, in the full IRAC fields of the 16 white dwarfs from Paper I, which shared $600 \mathrm{~s}$ integration times per filter, and identical 20-point dithering patterns. These sources were successfully detected and extracted photometrically by the IRAF tasks daofind and daophot. Based on the number of detections per magnitude bin (disregarding any trends in the number of source counts as a function of wavelength), it is clear that the $7.9 \mu \mathrm{m}$ channel would limit any four-channel IRAC survey for objects whose spectral energy distributions are not rising toward longer wavelengths.

\subsubsection{Detectability of T and Sub-T Dwarfs}

There exist a total of 58 white dwarfs which have been observed with IRAC at $7.9 \mu \mathrm{m}$ utilizing a common experimental design: 22 targets from the present work, 16 white dwarfs from Paper I, and 10 degenerates from Jura et al. (2007a). This white dwarf data set allows an empirical assessment of the photometric sensitivity at this longest wavelength, and contains 35 unambiguous detections in that channel, with point sources as faint as $0.06 \mathrm{mJy}$ reliably detected in all backgrounds. This finding is consistent with: (1) the published sensitivities for IRAC (Spitzer Science Center 2006b), (2) calculations by the Sensitivity Performance Estimation Tool, and (3) the number of sources detected as a function of magnitude in Figure 9. Therefore at $m_{7.9 \mu \mathrm{m}}=$ $15.0 \mathrm{mag}$, or $0.064 \mathrm{mJy}$, point sources should be well detected regardless of background.

The T dwarf sequence down to spectral type T8 ends at $M_{7.9 \mu \mathrm{m}}=13.3 \mathrm{mag}$ (Patten et al. 2006). This corresponds to $m_{7.9 \mu \mathrm{m}}=14.8 \mathrm{mag}$ at a distance of $20 \mathrm{pc}$, and thus any widely separated T dwarf companions to the $d \leq 20$ pc targets should be readily detected in this channel. Similar calculations in the other three IRAC channels estimate that a T8 dwarf should be correspondingly well detected out to: $20 \mathrm{pc}$ at $5.7 \mu \mathrm{m}, 60 \mathrm{pc}$ at $3.6 \mu \mathrm{m}$, and $100 \mathrm{pc}$ at $4.5 \mu \mathrm{m}$ (Patten et al. 2006). For the white 
TABLE 5

Target Ages and Upper-Mass Limits for Unresolved Companions

\begin{tabular}{rccccrr}
\hline \hline WD Number & $\begin{array}{c}t_{\mathrm{ms}} \\
(\mathrm{Gyr})\end{array}$ & $\begin{array}{c}t_{\mathrm{wd}} \\
(\mathrm{Gyr})\end{array}$ & $\begin{array}{c}\tau^{\mathrm{a}} \\
(\mathrm{Gyr})\end{array}$ & $\begin{array}{c}d \\
(\mathrm{pc})\end{array}$ & $\begin{array}{c}M_{4.5 \mu \mathrm{m}} \\
(\mathrm{mag})\end{array}$ & $\begin{array}{r}\text { Mass } \\
\left(M_{\mathrm{J}}\right)\end{array}$ \\
\hline $0349+247 \ldots \ldots \ldots$. & $\ldots$ & $\ldots$ & 0.1 & 132 & 12.3 & 9 \\
$0352+096 \ldots \ldots \ldots$. & $\ldots$ & $\ldots$ & 0.6 & 46 & 13.7 & 10 \\
$0406+169 \ldots \ldots \ldots$. & $\ldots$ & $\ldots$ & 0.6 & 46 & 14.4 & 7 \\
$0415+271 \ldots \ldots \ldots$. & $\ldots$ & $\ldots$ & 0.6 & 46 & 13.9 & 9 \\
$0421+162 \ldots \ldots \ldots \ldots$ & $\ldots$ & $\ldots$ & 0.6 & 46 & 13.7 & 10 \\
$0425+168 \ldots \ldots \ldots$. & $\ldots$ & $\ldots$ & 0.6 & 46 & 13.6 & 10 \\
$0431+126 \ldots \ldots \ldots$. & $\ldots$ & $\ldots$ & 0.6 & 46 & 13.6 & 10 \\
$0437+138 \ldots \ldots \ldots$. & $\ldots$ & $\ldots$ & 0.6 & 46 & 14.2 & 8 \\
$0438+108 \ldots \ldots \ldots \ldots$. & $\ldots$ & $\ldots$ & 0.6 & 46 & 13.4 & 11 \\
\hline
\end{tabular}

\begin{tabular}{|c|c|c|c|c|c|c|}
\hline \multicolumn{7}{|c|}{ Young Field White Dwarfs } \\
\hline $0001+433 \ldots \ldots \ldots \ldots$ & $\ldots$ & $\ldots$ & 0.1 & 96 & 12.4 & 9 \\
\hline $0136+251 \ldots \ldots \ldots \ldots$ & $\ldots$ & $\ldots$ & 0.1 & 73 & 13.0 & 6 \\
\hline $0235-125 \ldots \ldots \ldots$ & $\ldots$ & $\ldots$ & 0.1 & 65 & 13.3 & 5 \\
\hline $0236+498 \ldots \ldots \ldots \ldots$ & 0.1 & 0.1 & 0.2 & 107 & 11.9 & 11 \\
\hline 0325-857A ......... & 0.1 & 0.3 & 0.4 & 35 & 13.9 & 6 \\
\hline $0325-857 \mathrm{~B} \ldots \ldots \ldots$ & $\ldots$ & $\ldots$ & 0.4 & 35 & 14.5 & 5 \\
\hline $0346-011 \ldots \ldots \ldots . .$. & $\ldots$ & $\ldots$ & 0.1 & 30 & 14.5 & 3 \\
\hline $0440-038 \ldots \ldots \ldots . .$. & $\ldots$ & $\ldots$ & 0.1 & 134 & 11.9 & 10 \\
\hline $0518-105 \ldots \ldots \ldots$. & $\ldots$ & $\ldots$ & 0.1 & 92 & 12.5 & 8 \\
\hline $0531-022 \ldots \ldots \ldots$. & 0.1 & 0.1 & 0.2 & 107 & 12.6 & 9 \\
\hline $0652-563 \ldots \ldots \ldots$. & $\ldots$ & $\ldots$ & 0.1 & 119 & 11.7 & 11 \\
\hline $0730+487 \ldots \ldots \ldots \ldots$ & 0.1 & 0.4 & 0.5 & 39 & 14.5 & 6 \\
\hline $0821-252 \ldots \ldots \ldots$. & $\ldots$ & $\ldots$ & 0.1 & 105 & 12.2 & 10 \\
\hline $0914-195 \ldots \ldots \ldots . .$. & $\ldots$ & $\ldots$ & 0.1 & 175 & 11.3 & 12 \\
\hline $1022-301 \ldots \ldots \ldots$. & $\ldots$ & $\ldots$ & 0.1 & 61 & 13.4 & 5 \\
\hline $1440+753 \ldots \ldots \ldots$. & $\ldots$ & $\ldots$ & 0.1 & 101 & 12.7 & 7 \\
\hline $1529-772 \ldots \ldots \ldots . .$. & $\ldots$ & $\ldots$ & 0.1 & 137 & 12.3 & 9 \\
\hline $1543-366 \ldots \ldots \ldots$ & $\ldots$ & $\ldots$ & 0.1 & 111 & 12.5 & 8 \\
\hline $1609+631 \ldots \ldots \ldots . .$. & $\ldots$ & $\ldots$ & 0.1 & 134 & 12.6 & 8 \\
\hline $1642+413 \ldots \ldots \ldots \ldots$ & 0.1 & 0.1 & 0.2 & 109 & 12.8 & 8 \\
\hline $1658+440 \ldots \ldots \ldots \ldots$ & $\ldots$ & $\ldots$ & 0.1 & 32 & 14.8 & 2 \\
\hline $1740-706 \ldots \ldots \ldots$ & $\ldots$ & $\ldots$ & 0.1 & 76 & 13.5 & 5 \\
\hline \multicolumn{7}{|c|}{ Metal-Rich Field White Dwarfs } \\
\hline $0032-175 \ldots \ldots \ldots$ & 1.5 & 0.7 & 2.2 & 31 & 13.9 & 18 \\
\hline $0046+051 \ldots \ldots \ldots . .$. & 0.2 & 3.7 & 3.9 & 4.4 & 15.2 & 13 \\
\hline $0235+064 \ldots \ldots \ldots \ldots$ & 1.2 & 0.2 & 1.4 & 70 & 13.3 & 20 \\
\hline $0322-019 \ldots \ldots \ldots$. & 1.2 & 4.4 & 5.6 & 17 & 15.1 & 17 \\
\hline $0846+346 \ldots \ldots \ldots \ldots$ & 1.9 & 1.4 & 3.3 & 30 & 14.6 & 17 \\
\hline $1102-183 \ldots \ldots \ldots$. & 1.5 & 1.1 & 2.6 & 40 & 14.2 & 17 \\
\hline $1124-293 \ldots \ldots \ldots . .$. & 0.9 & 0.6 & 1.5 & 34 & 14.3 & 12 \\
\hline $1204-136 \ldots \ldots \ldots$ & 1.5 & 0.4 & 1.9 & 62 & 13.5 & 20 \\
\hline $1208+576 \ldots \ldots \ldots$ & 4.0 & 2.5 & 6.5 & 20 & 14.7 & 25 \\
\hline $1344+106 \ldots \ldots \ldots . .$. & 0.7 & 1.9 & 2.6 & 20 & 14.7 & 14 \\
\hline $1407+425 \ldots \ldots \ldots \ldots$ & 0.3 & 0.9 & 1.2 & 33 & 14.6 & 10 \\
\hline $1455+298 \ldots \ldots \ldots . .$. & 2.4 & 1.4 & 3.8 & 36 & 14.1 & 25 \\
\hline $1632+177 \ldots \ldots \ldots$ & 2.4 & 0.6 & 3.0 & 16 & 14.1 & 20 \\
\hline $1633+433 \ldots \ldots \ldots \ldots$ & 0.3 & 2.7 & 3.0 & 15 & 14.8 & 14 \\
\hline $1826-045 \ldots \ldots \ldots$ & 3.0 & 0.8 & 3.8 & 29 & 13.0 & 35 \\
\hline $1858+393 \ldots \ldots \ldots$ & 1.5 & 0.7 & 2.2 & 45 & 14.2 & 16 \\
\hline
\end{tabular}

a The total age of the Pleiades white dwarf EG 25 (Table 1) and the similarly hot and massive field white dwarfs ( Table 2 ) is taken to be $0.125 \mathrm{Gyr}(\operatorname{see} \S 4.1$ ). The last column lists the companion-mass upper limit.

dwarf targets closer than $20 \mathrm{pc}$, the IRAC observations should be sensitive to a small range of substellar companions with $M_{7.9 \mu \mathrm{m}}>$ $13.3 \mathrm{mag}$, and potentially of a later spectral type than $\mathrm{T}$.

Owing to the nature of the dithering pattern, a further assessment must be made in regards to the effective field of view for
TABLE 6

Target Ages and Upper-Mass Limits for Resolved Companions

\begin{tabular}{|c|c|c|c|c|c|c|}
\hline WD Number & $\begin{array}{c}t_{\mathrm{ms}} \\
(\mathrm{Gyr})\end{array}$ & $\begin{array}{c}t_{\mathrm{wd}} \\
(\mathrm{Gyr})\end{array}$ & $\begin{array}{c}\tau \\
(\mathrm{Gyr})\end{array}$ & $\begin{array}{c}d \\
(\mathrm{pc})\end{array}$ & $\begin{array}{c}M_{7.9 \mu \mathrm{m}} \\
(\mathrm{mag})\end{array}$ & $\begin{array}{l}\text { Mass } \\
\left(M_{\mathrm{J}}\right)\end{array}$ \\
\hline $0046+051$ & 0.2 & 3.7 & 3.9 & 4.4 & 16.0 & 25 \\
\hline $0322-019$.................... & 1.2 & 4.4 & 5.6 & 17 & 13.8 & 65 \\
\hline $1208+576$ & 4.0 & 2.5 & 6.5 & 20 & 13.5 & 70 \\
\hline $1344+106 \ldots \ldots \ldots \ldots \ldots$ & 0.7 & 1.9 & 2.6 & 20 & 13.5 & 60 \\
\hline $1632+177$..................... & 2.4 & 0.6 & 3.0 & 16 & 14.0 & 50 \\
\hline $1633+433$ & 0.3 & 2.7 & 3.0 & 15 & 14.1 & 50 \\
\hline $2326+049$ & 0.4 & 0.5 & 0.9 & 14 & 14.3 & 25 \\
\hline
\end{tabular}

Note.-The exposure time for $0046+051$ was $150 \mathrm{~s}$ vs. $600 \mathrm{~s}$ for the other targets, resulting in an overall sensitivity about half of that calculated in $\S 4.3 .1$.

the depth described above. For all but vMa 2, the medium-sized cycling pattern was used, which should result in an effective coverage equal to the IRAC field of view minus about 25 pixels at each edge, or approximately $4.1^{\prime} \times 4.1^{\prime}$, consistent with the analyzed images. For vMa 2, the effective field of view is about 12 pixels larger at each edge, or approximately $4.6^{\prime} \times 4.6^{\prime}$, and the sensitivity was $0.75 \mathrm{mag}$ less at each channel owing to a $150 \mathrm{~s}$ total integration time.

\subsubsection{Selection of T and Sub-T Dwarfs}

For each white dwarf searched for wide $\mathrm{T}$ and sub-T dwarf companions, all four IRAC filter images were aligned and combined to create a single master coordinate image. The IRAF task daof ind was executed on this master IRAC image to select point sources with counts at or above $3 \sigma$, and the resulting coordinate list was then fed into daophot for each filter image in order to perform automated point-spread function fitting photometry.

Template point-spread functions were created by running daophot on IRAC images of the Galactic component of the Spitzer Galactic First Look Survey to select approximately half to one dozen bright, unsaturated point sources in each filter. The magnitudes of these selected template sources were calculated by creating zero points which included the zero magnitude fluxes for IRAC, multiplication by the appropriate unit area on the array, and the necessary conversion of units. If the standard $r=10$ pixel radius aperture is used for photometry, these zero points are $(17.30,16.81,16.33$, and 15.69) $\mathrm{mag}$ at $(3.6,4.5,5.7$, and 7.9$) \mu \mathrm{m}$.

The extracted sources in each filter were cross-correlated using the master coordinate list, and two color-color diagrams were generated from the results: $m_{3.6 \mu \mathrm{m}}-m_{4.5 \mu \mathrm{m}}$ versus $m_{5.7 \mu \mathrm{m}}-$ $m_{7.9 \mu \mathrm{m}}$, and $m_{3.6 \mu \mathrm{m}}-m_{4.5} \mu \mathrm{m}$ versus $m_{4.5 \mu \mathrm{m}}-m_{5.7 \mu \mathrm{m}}$. In these planes, T and sub-T dwarf candidates were selected by demanding an object meet three criteria suggested by the IRAC colors

TABLE 7

Representative $4.5 \mu \mathrm{m}$ Absolute Magnitudes for Substellar Objects

\begin{tabular}{|c|c|c|c|c|}
\hline $\begin{array}{l}\text { Mass } \\
\left(M_{\mathrm{J}}\right)\end{array}$ & $0.1 \mathrm{Gyr}$ & $0.5 \mathrm{Gyr}$ & $1.0 \mathrm{Gyr}$ & $5.0 \mathrm{Gyr}$ \\
\hline 2 & 14.8 & 16.7 & 17.7 & 21.1 \\
\hline 5 & 13.2 & 14.9 & 15.7 & 17.9 \\
\hline $10 \ldots \ldots \ldots$ & 12.0 & 13.5 & 14.3 & 16.1 \\
\hline $15 \ldots \ldots \ldots \ldots \ldots \ldots \ldots$ & 11.1 & 12.7 & 13.5 & 15.3 \\
\hline $20 \ldots \ldots \ldots \ldots \ldots \ldots \ldots \ldots .$. & 10.9 & 12.2 & 12.9 & 14.7 \\
\hline
\end{tabular}

Notes.-Entries are in magnitudes (Spitzer Science Center 2006a). The table is based on the models of I. Baraffe (2007, private communication; Baraffe et al. 2003). 

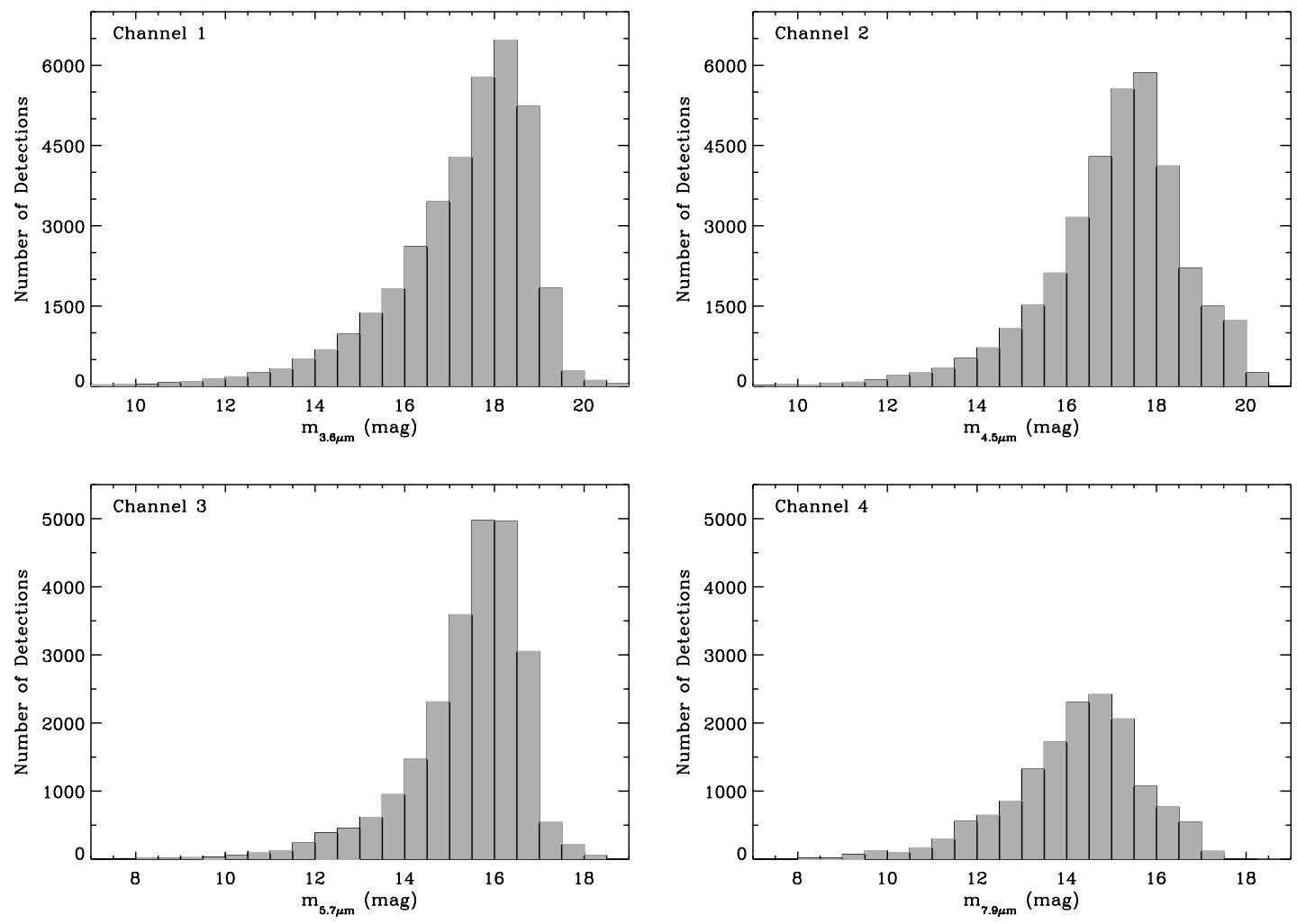

FIG. 9.- Sum of the number of pointlike source detections as a function of magnitude at each wavelength in the IRAC imaged fields of 16 metal-rich white dwarfs with $600 \mathrm{~s}$ exposure times (Paper I).

presented in Patten et al. (2006): $0.2<m_{3.6 \mu \mathrm{m}}-m_{4.5} \mu \mathrm{m}<3.0$, $0.0<m_{5.7 \mu \mathrm{m}}-m_{7.9 \mu \mathrm{m}}<1.5$, and $-1.5<m_{4.5 \mu \mathrm{m}}-m_{5.7 \mu \mathrm{m}}<$ 1.0 , with error bars ignored in the first cut. All objects thus selected were examined individually: their images inspected and photometric data further evaluated. Although the mid-infrared colors of sub-T type objects are somewhat uncertain, model predictions yield colors which fit with the selection criteria above (D. Saumon 2006, private communication). Unfortunately, the earliest T dwarfs do not stand out strongly in IRAC color-color diagrams, and require near-infrared photometry to be clearly distinguished (Patten et al. 2006).

No candidates which met all the criteria and passed critical examination were found in the IRAC fields of the seven white dwarfs within $d=20 \mathrm{pc}$. While testing the color-color selection and extraction algorithm, the procedure was conducted at all 17 metal-rich white dwarfs in Table 3. A typical detection which met the color criteria had one or more of the following problems: (1) large photometric errors, (2) a location near the noisy edge of the image, (3) probable confusion with another source, (4) association with a known image artifact, and (5) a substantially discrepant color-magnitude relation at the expected distance. Less often a detection would be a very red, unresolved extragalactic source whose nature was confirmed via existing optical astrometric and photometric catalogs. Table 6 lists the resulting upper limits on substellar mass companions achieved using this method, calculated by transforming $m_{7.9 \mu \mathrm{m}}=15.0 \mathrm{mag}$ to the expected $M_{7.9 \mu \mathrm{m}}$ at the target distance, then using substellar cooling models to obtain a mass from this flux at the appropriate total age (I. Baraffe 2007, private communication; Baraffe et al. 2003).

To test the selection and extraction algorithm, the IRAC fields of three T dwarfs were downloaded from the Spitzer archive (program 35; PI: G. G. Fazio) and the procedure was run on images containing objects with spectral types T2.0, T5.0, and
T8.0 (Patten et al. 2006). All three objects were selected correctly by the color-color cuts, and with modest photometric error bars indicating genuine detections. There is a nonzero probability that a bona fide cold brown dwarf companion escaped detection among our white dwarf target fields, despite the ability of daophot to spatially and photometrically deconvolve overlapping point sources. Based on the detection logs and the statistics from Figure 9, in the two short-wavelength IRAC filter images there were roughly 1000 sources per field with brightnesses greater than the completeness limit in those bandpasses. Taking a worst-case scenario in which all these sources represent potential spoilers yields a $2 \%$ probability of chance alignment within the relatively large, reduced image field of view.

$$
\text { 4.3.3. The Hyades }
$$

Figure 10 plots color-magnitude diagrams for all detected pointlike sources in the fields of the six Hyades white dwarfs observed in both of the short-wavelength IRAC channels. Included in the plot is the expected T dwarf sequence (T1-T8; Patten et al. 2006) at the $46 \mathrm{pc}$ distance to the open cluster. The cooler part of the $\mathrm{T}$ dwarf sequence appears to stand out from most field objects. All sources with $m_{3.6 \mu \mathrm{m}}-m_{4.5 \mu \mathrm{m}}>1.0$ were investigated individually, revealing a few extragalactic sources, spurious detections near bright stars or the edge of the mosaic, and sources with large photometric errors. No reliable candidates near the T dwarf sequence were identified. Since $T$ and sub-T dwarfs should not be detected at the distance to the Hyades in the two long-wavelength IRAC channels, this limits what can be done with single-epoch IRAC data to constrain wide, methane-bearing substellar companions. Moreover, because very cool brown dwarfs will have $K-m_{3.6 \mu \mathrm{m}} \gtrsim 1$ (Patten et al. 2006), they should not be detected in the Two Micron All Sky Survey (2MASS), as any such companions in the Hyades would have $K \gtrsim 16$ mag. Therefore, further 

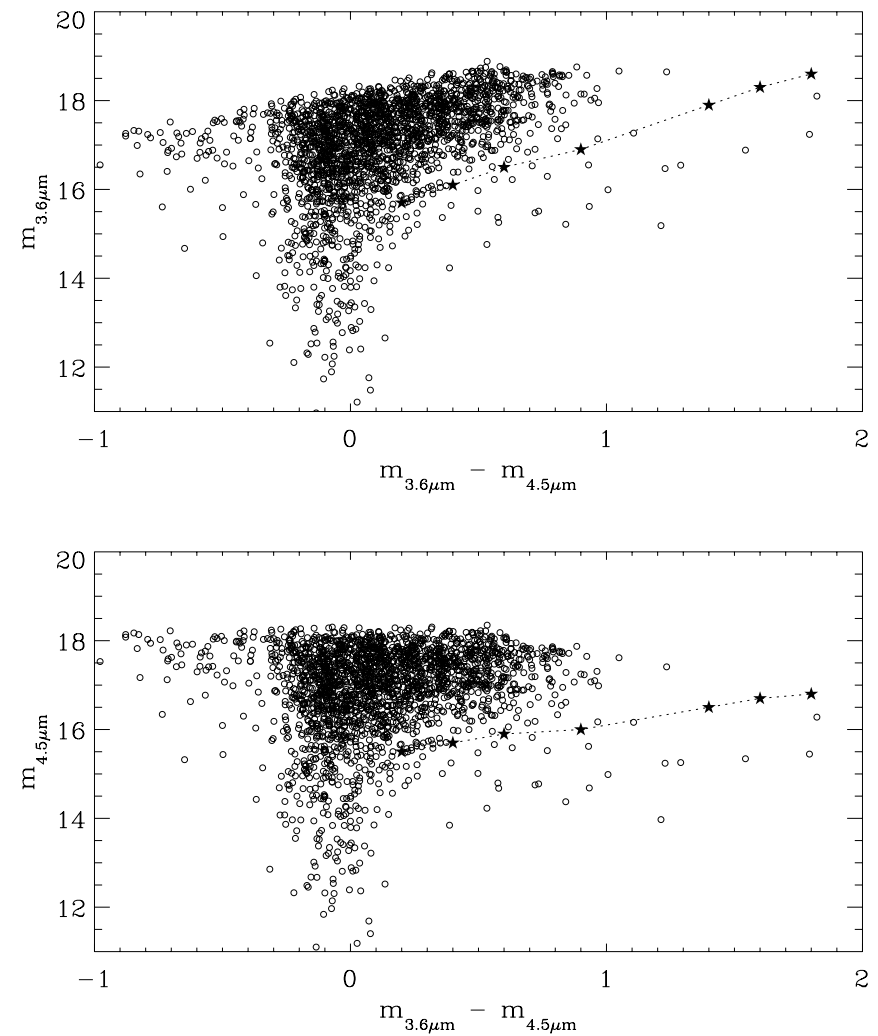

FIG. 10.-IRAC 3.6 and $4.5 \mu \mathrm{m}$ color-magnitude diagrams for all pointlike sources in the fields of the six Hyades white dwarfs observed at both short wavelengths. Also plotted (stars) are representative points in the IRAC T dwarf sequence (T1-T8; Patten et al. 2006) at the $46 \mathrm{pc}$ distance to the open cluster.

analysis of these data sets can only be achieved with deep nearinfrared imaging from the ground or proper-motion analysis with a second-epoch IRAC observation.

\section{LIMITS ON MASSIVE PLANETS AND COLD BROWN DWARF COMPANIONS}

Clearly, none of the 32 stars in Figures 1-8 display reliably measured photometric excess at $4.5 \mu \mathrm{m}$. When combined with similar results for the white dwarfs analyzed in Paper I, there are a total of 47 observed degenerates which reveal no evidence for unresolved, cold brown dwarf or massive planetary companions. This is a striking result, especially considering the large number of relatively young white dwarfs where massive planets would be detectable. There are 34 white dwarfs for which the IRAC $4.5 \mu \mathrm{m}$ observations were sensitive to unresolved planetary-mass companions in the range $2-13 M_{\mathrm{J}}$, and 10 white dwarfs for which the data were sensitive to brown dwarf companions in the range $14-20 M_{\mathrm{J}}$, according to substellar cooling models used here (I. Baraffe 2007, private communication; Baraffe et al. 2003).

\subsection{The Influence of Stellar Evolution on Massive Planets}

The aperture photometry and image analysis places limits on the presence of unresolved or partially resolved companions out to approximately 5 pixels or $6^{\prime \prime}$, the largest aperture used for photometry (Paper I). This angle on the sky corresponds to a several hundred AU region around the target white dwarfs, which lie at typical distances in the range $d \approx 20-100$ pc. However, any planets which formed in the 5-50 AU range during the mainsequence phase should now be located farther out due to orbital expansion via mass loss during the asymptotic giant branch
(Farihi et al. 2005b, 2006; Burleigh et al. 2002; Zuckerman \& Becklin 1987; Jeans 1924), yet still within a region to which the IRAC photometry is sensitive. While a typical expansion factor (equal to the ratio of the main-sequence progenitor mass to the white dwarf mass) is 2-3 for F-type stars, the massive young degenerates studied here are likely to produce much larger increases, up to a factor of 6 or so.

Tidal interactions should be relatively strong for massive planets orbiting intermediate-mass stars, whose radii can become as large as several AU at the tip of the asymptotic giant branch. For example, a $5 M_{\odot}$ main-sequence star should grow to a maximum radius near 5 AU (Villaver \& Livio 2007), directly engulfing any planets in that range. Using equation (6) of Rasio et al. (1996) for such a star, and assuming a convective envelope mass near $3 M_{\odot}$, a $10 M_{\mathrm{J}}$ planet at $10 \mathrm{AU}$ should tidally decay within the 1 Myr lifetime of its asymptotic giant branch host (Vassiliadis \& Wood 1993). Although this calculation is likely oversimplified, it is instructive; eschewing direct engulfment is not sufficient for a planet to survive the post-main sequence. Rather, the more massive the planet, the more it will induce tides in the giant star, and the more likely it will experience orbital decay and be destroyed (Rasio et al. 1996). One competing factor is the fact that by the time an asymptotic giant has reached it maximum radius, there has been significant mass lost and the orbits of any planets should already have expanded commensurately. However, a complete, time-dependent treatment of these competing forces - orbital expansion due to mass loss versus orbital decay due to tidal forces - on planets during the asymptotic giant branch has not been carried out. Such a study would be highly valuable, but is beyond the scope of this paper.

\subsection{Massive Planets and Brown Dwarfs at Evolved Stars}

Recent work indicates a substantial percentage of substellar, radial velocity companions to first-ascent giant stars are potentially or certainly above the deuterium burning minimum mass. Presently four of 13 or $31 \%$ of known substellar companions to giant stars have masses above $13 M_{\mathrm{J}}$ (Liu et al. 2008; Lovis \& Mayor 2007; Niedzielski et al. 2007; Hatzes et al. 2005). Two items of interest for white dwarf planetary system studies emerge from these results at giant stars. First, the $M \sin i$ distribution of close substellar companions to intermediate-mass, evolved stars is markedly different than for solar-type main-sequence stars. Second is the fact that all four brown dwarf hosting giants have $2 M_{\odot}<M<4 M_{\odot}$, and are thus related to the population of white dwarfs studied in this paper.

With orbital semimajor axes 0.5 AU $<a<2.5$ AU (Liu et al. 2008; Lovis \& Mayor 2007; Niedzielski et al. 2007), all of the known substellar radial velocity companions to first-ascent giant stars risk destruction during the ensuing asymptotic giant phase. However, the most apparently brown dwarf-like companions have $M \sin i \approx 20 M_{\mathrm{J}}$ (Liu et al. 2008; Lovis \& Mayor 2007): potentially massive enough to eject the giant stellar envelope as have the 50-60 $M_{\mathrm{J}}$ substellar companions to the white dwarfs WD 0137-049 and GD 1400 (Burleigh et al. 2006; Maxted et al. 2006; Farihi et al. 2005b; Farihi \& Christopher 2004). The close, $P \approx 2 \mathrm{hr}$ substellar companion to WD $0137-049$ is thought to have ejected the dense first-ascent giant envelope of its host, as evidenced by the fact the white dwarf is a low-mass, helium core degenerate. This is not the case for GD 1400B, whose degenerate host is a typical, carbon-oxygen core white dwarf, but its $P \approx$ $10 \mathrm{hr}$ period (M. R. Burleigh et al. 2008, in preparation) indicates likely prior orbital decay due to the ejection of the asymptotic giant envelope. By inference, both these substellar 
survivors would have originally orbited within roughly $1 \mathrm{AU}$ of their host intermediate-mass stellar progenitors.

\subsection{Formation, Persistence, and Sacrifice}

The results of this IRAC white dwarf study may imply that close massive planetary and brown dwarf companions to intermediate-mass stars do not typically survive the asymptotic giant branch. Robust statistics are not yet available, but Lovis \& Mayor (2007) estimate at least 3\% of stars with $M \gtrsim 1.8 M_{\odot}$ mass stars host $M \sin i>5 M_{\mathrm{J}}$ companions, and as discussed in $\S 5.2$, about $1 / 3$ of these are brown dwarfs (according to the IAU definition). Hence, a reasonably optimistic expectation for an IRAC search of 46 white dwarfs would be one or two detections. This negative result suggests the possibility that a higher (substellar) companion mass is required to survive the entire post-main sequence-specifically the asymptotic giant phase - at orbital separations of a few to several AU.

However, recent evidence has suggested a mechanism which may allow planets to survive within the inner regions through both giant phases: sacrifice of the innermost components. Analogous to the formation of several types of post-main-sequence binaries, substellar companions can dynamically eject the (first or second phase) giant envelope (Nelemans \& Tauris 1998; Soker 1998), thereby shielding any remaining components in a planetary system. This process - common envelope evolution - is responsible for the close orbits of numerous low-mass stellar and substellar companions to white dwarfs (Farihi et al. 2006; Schreiber \& Gänsicke 2003). Essentially, unstable mass transfer from the giant results in a frictional exchange of orbital energy between the secondary and the envelope material, resulting in an orbit decrease and an efficient envelope ejection (Paczynski 1976). The $M \sin i \approx$ $3 M_{\mathrm{J}}, a=1.7$ AU planet recently detected at the sdB star V391 Pegasi (Silvotti et al. 2007) has survived a first-ascent giant phase involving significant mass loss, possibly due to such an interaction (Han et al. 2002; Nelemans \& Tauris 1998; Soker 1998). Similarly, the $M \sin i \approx 2 M_{\mathrm{J}}, a=2.5$ AU planet candidate at the pulsating white dwarf GD 66 (Mullally et al. 2008) may owe its inner region survival to the sacrifice of closer planets.

While intermediate separation planets may have indeterminate fates, any massive planets originally orbiting at $a \gtrsim 5 \mathrm{AU}$ should now be located at tens to hundreds of AU in the white dwarf phase, having essentially eluded any substantial post-mainsequence interaction with their host star (Villaver \& Livio 2007). The results of this IRAC search imply that massive, $M \gtrsim 10 M_{\mathrm{J}}$ planets and brown dwarfs form rarely $(f \lesssim 3 \%$ ) at these wide separations.

\subsection{Planets in the Hyades}

The massive planet recently found at the Hyades giant $\epsilon$ Tauri (Sato et al. 2007) is very interesting in light of the seven classical Hyades white dwarfs surveyed with IRAC — would such a planet have been detected if it persisted into the white dwarf evolutionary phase? The most likely mass of the planet at $\epsilon$ Tauri is near $10 M_{\mathrm{J}}$, and the IRAC $4.5 \mu \mathrm{m}$ photometry was sensitive to objects of this mass at virtually every Hyades target, although in reality such a detection might prove more or less difficult due to differences from the model predictions used here. However, it is uncertain whether this Hyades planet at $a=1.9 \mathrm{AU}$ will survive the asymptotic giant phase of its host, since the maximum radius of the star will reach at least $3 \mathrm{AU}$ (Villaver \& Livio 2007).

A clear advantage of the IRAC search of the Hyades white dwarfs is insensitivity to orbital separation or inclination, pa- rameter spaces which limit radial velocity, and direct imaging searches for planets. The resulting substellar mass sensitivities achieved here for the Hyades are comparable to those produced via direct imaging with the Hubble Space Telescope NearInfrared Camera and Multi-Object Spectrometer (NICMOS)also around $10 M_{\mathrm{J}}$ (Friedrich et al. 2005)—but for any orbits out to $a \approx 250$ AU. Only very widely separated massive planets should have escaped detection.

\subsection{Cold Brown Dwarfs}

The previous sections have covered discussions of mass, but not of temperature. For 27 targets in the survey, the IRAC $4.5 \mu \mathrm{m}$ photometry was sensitive to the entire known $\mathrm{T}$ dwarf sequence, independent of the corresponding masses, and in a few cases more than a full magnitude fainter than a T8 dwarf (Patten et al. 2006). In fact, with the exception of a single target (G21-16; see $\S 5.7$ ) whose IRAC image was confused with other sources, this survey rules out brown dwarf companions down to $25 M_{\mathrm{J}}$ within a few hundred AU of all white dwarf targets, implying a brown dwarf companion fraction less than around $2 \%$. If the minimal sensitivity achieved here for these 46 targets is matched by similar IRAC $4.5 \mu \mathrm{m}$ results for 124 nearby white dwarfs (Mullally et al. 2007), this fraction could be smaller than $0.6 \%$, and consistent with previous white dwarf studies which were sensitive to somewhat higher temperatures and masses (L dwarfs; Hoard et al. 2007; Farihi et al. 2005a; Farihi 2004; Wachter et al. 2003; Zuckerman \& Becklin 1987).

\subsection{No Evidence for Merger Products}

Although not the focus of this study, the results give no indication of lasting merger products at any of the 10 additional massive white dwarfs observed specifically for this study. Combined with the results of Hansen et al. (2006), there is as yet no indication of disks (and subsequent pollution) or reforged planets at nearly two dozen massive white dwarfs which could conceivably have formed as a merger of lower mass degenerates (Liebert et al. 2005a). Given the recent results on externally polluted white dwarfs with debris disks, it is possible that any dust disks formed via white dwarf mergers would dissipate rapidly, the particles becoming gaseous through mutual collisions within the disk (Paper I).

\subsection{Individual Objects}

$0046+051$ (vMa 2). - At $4.4 \mathrm{pc}$, this degenerate represents a unique and advantageous hunting ground for planets and planetary system remnants. This cool, helium atmosphere, metal-rich white dwarf has been externally polluted by interstellar or circumstellar matter. Previous ground- and space-based mid-infrared imaging and photometry have ruled out the presence of a substellar companion suggested by Makarov (2004) down to $T_{\text {eff }} \gtrsim$ $500 \mathrm{~K}$ and corresponding to around $25 M_{\mathrm{J}}$ at $5 \mathrm{Gyr}$ (Farihi et al. 2004; Burrows et al. 2003). The IRAC $4.5 \mu \mathrm{m}$ photometry of vMa 2 and the models used here rule out the presence of a companion as cool as $400 \mathrm{~K}$, and a mass close to the deuterium burning limit at 4.4 Gyr. Furthermore, the IRAC four-channel color-color search for resolved substellar companions rules out the presence of any widely bound object as cool as $T_{\text {eff }} \approx 550 \mathrm{~K}$ within $r \approx 1200 \mathrm{AU}$ of vMa 2 . Models predict this should correspond to a mass of $25 M_{\mathrm{J}}$ at the age of this well-studied white dwarf.

Deep, ground-based, $J$-band imaging observations have ruled out widely bound planetary-mass companions to vMa 2 as small as $7 M_{\mathrm{J}}$ at orbital separations near 10-200 AU (Burleigh et al. 2008). There exist two epochs of IRAC $4.5 \mu \mathrm{m}$ imaging of 
vMa 2 in the Spitzer archive, separated by $2.1 \mathrm{yr}$ and clearly revealing $6.2^{\prime \prime}$ of proper motion on blinking the aligned frames. There are no field objects comoving with the white dwarf, ruling out well-detected, resolved objects with $m_{4.5} \mu \mathrm{m}=16.7 \mathrm{mag}$ (see Fig. 9) or $36 \mu \mathrm{Jy}$ as companions within $1200 \mathrm{AU}$ of vMa 2. At the $4.4 \mathrm{pc}$ distance and $4.4 \mathrm{Gyr}$ age of vMa2, models predict that this very sensitive observational limit corresponds to a mass of $4 M_{\mathrm{J}}$ and a temperature of just $T_{\mathrm{eff}} \approx 200 \mathrm{~K}$ : significantly lower than the known $\mathrm{T}$ dwarfs and only $40 \mathrm{~K}$ warmer than Jupiter(!).

0236+498 (EUVE J0239+50.0).- - Very little reliable photometry exists on this hot and faint degenerate, making it difficult to properly calibrate its spectral energy distribution (see Fig. 2). The 2MASS $J$-band flux is likely the most reliable photometric data available, while the $H$-band flux has a large associated error. The white dwarf is clearly detected in the short-wavelength IRAC images, but significant uncertainty exists at the long wavelengths, where the flux in the photometric aperture could be due to a background source or noise. Unfortunately, complications plague the short-wavelength IRAC observations: a column pull-down artifact at the position of the white dwarf and a photometrically overlapping point source located 3.6" away. The IRAF routine daophot was used to deconvolve the flux of the white dwarf and the nearby point source, after manually correcting the column pull-down artifact by adding the median value of nearby columns. Despite these efforts, it is possible or even likely the photometry of the white dwarf is contaminated. If the 2MASS $H$-band flux and IRAC photometry are somewhat accurate, then the white dwarf may possess an excess. Ground-based near-infrared photometry is needed for this source.

0325-857AB ( $L B$ 9802AB).-This visual pair has long been a binary suspect (Hansen et al. 2006; Barstow et al. 1995), but its physical companionship is confirmed here for the first time. Previous arguments for binarity relied on space density, which is insufficient to firmly establish companionship, especially in a rare system such as this, where the more massive of the pair white dwarfs is the hotter and apparently younger star. Common proper motion is demonstrated by measuring the astrometric offsets of the white dwarfs and 20 background point sources between two epochs of SuperCOSMOS data. Two scans of UKST photographic plates were used for this purpose, one image taken in 1976.7 and another from 2001.9, providing a $25.3 \mathrm{yr}$ baseline. Using the IRAF routine geomap to measure both the motion of the white dwarfs and the random centroiding errors from the positions of the background sources, the component proper motions are: $\mu_{A}=0.074 \operatorname{arcsec}_{\mathrm{yr}^{-1}}$ at $\theta_{A}=244^{\circ}$ and $\mu_{B}=$ $0.083 \operatorname{arcsec} \mathrm{yr}^{-1}$ at $\theta_{B}=242^{\circ}$, with $\sigma_{\mu}=0.012 \operatorname{arcsec} \mathrm{yr}^{-1}$ and $\sigma_{\theta}=6^{\circ}$. The separation between primary and secondary from the 2MASS $J$-band image is $6.9^{\prime \prime}$ at a position angle of $325^{\circ}$.

$1455+298$ (G166-58).--This metal-rich white dwarf displays excess flux in the two long-wavelength IRAC channels, but not at $4.5 \mu \mathrm{m}$ (Paper I). Therefore, it was included in the photometric analysis for unresolved substellar companions.

$2326+049$ (G29-38).-Not included in the analysis for unresolved companions due to its large photometric excess at all IRAC wavelengths (Paper I). However, the field surrounding G29-38 was searched for widely bound, cold substellar com- panions using the method described in $\S 4.3$, revealing no candidates down to $25 M_{\mathrm{J}}$ within $3500 \mathrm{AU}$.

\section{CONCLUSION}

A relatively comprehensive look at both young and older white dwarfs with IRAC reveals no promising evidence for massive planets or cold brown dwarf companions at orbital separations within a few hundred AU. By conducting a search for substellar companions via excess emission in the mid-infrared, the data cover the widest range of orbital phase space possible: nearly all possible semimajor axes, eccentricities, and inclinations, including the missed middle ground (5-50 AU) between radial velocity and direct imaging searches. The uncovered range of possible orbits lies roughly beyond a few hundred AU.

While it is somewhat uncertain how substellar objects evolve (dynamically or otherwise) within 5-10 AU of mass losing asymptotic giant stars, avoidance of direct engulfment may not suffice for ultimate survival to the white dwarf phase. However, massive inner planets could act as sacrificial guardians for remaining outer planetary system components. Any planets and brown dwarfs outside of this region should be relatively unaffected, and the IRAC results demonstrate a dearth of cold substellar companions down into sub-T dwarf temperatures. The $2-3 M_{\mathrm{J}}$ planets suspected at the sdB star V391 Pegasi and the white dwarf GD 66 are just beyond the reach of this survey.

The negative results for the Hyades and comparably aged white dwarfs yield upper-mass limits at or somewhat below $10 M_{\mathrm{J}}$ for objects which may have formed around $3 M_{\odot}$ main-sequence stars. Similar limits at the Pleiades and analogously hot and massive field white dwarfs provide the first evidence that massive planets are not commonly formed or do not survive the post-mainsequence evolution of intermediate-mass stars with $M \gtrsim 4 M_{\odot}$. This latter result was only achievable via observations of white dwarfs, as their main-sequence, B-type progenitors are not amenable to other planet detection techniques.

The lack of $4.5 \mu \mathrm{m}$ excess at all white dwarf targets, especially when combined with similar IRAC searches (Mullally et al. 2007), confirms that L- and T-type brown dwarf companions are rare $(f<0.6 \%)$ within a few hundred AU, down to masses near the deuterium burning limit. These results suggest the possibility that the lowest mass companions, and especially planets, orbiting intermediate-mass stars may be altered or destroyed prior to or during the post-main sequence, or are (more likely) too cold to directly detect with current facilities.

The authors are grateful to referee F. Mullally for comments which resulted in an improved manuscript. J. Farihi thanks M. Jura for helpful discussions on post-main-sequence evolution. This work is based on observations made with the Spitzer Space Telescope, which is operated by the Jet Propulsion Laboratory, California Institute of Technology, under a contract with NASA. Support for this work was provided by NASA through an award issued by JPL/Caltech, and by NASA grants to UCLA.

Facility: Spitzer (IRAC)
Baraffe, I., Chabrier, G., Barman, T. S., Allard, F., \& Hauschildt, P. H. 2003, A\&A, 402, 701

Barstow, M. A., Bond, H. E., Holberg, J. B., Burleigh, M. R., Hubeny, I., \& Koester, D. 2005, MNRAS, 362, 1134

Barstow, M. A., Jordan, S., O’Donoghue, D., Burleigh, M. R., Napiwotzki, R., \& Harrop-Allin, M. K. 1995, MNRAS, 277, 971

Becklin, E. E., \& Zuckerman, B. 1988, Nature, 336, 656

\section{REFERENCES}

Bergeron, P., Fontaine, G., Billeres, M., Boudreault, S., \& Green, E. M. 2004, ApJ, 600, 404

Bergeron, P., Leggett, S. K., \& Ruiz, M. T. 2001, ApJS, 133, 413 Bergeron, P., Liebert, J., \& Fullbright, M. S. 1995a, ApJ, 444, 810 Bergeron, P., Ruiz, M. T., \& Leggett, S. K. 1997, ApJS, 108, 339 Bergeron, P., Saffer, R. A., \& Liebert, J. 1992, ApJ, 394, 228 Bergeron, P., Saumon, D., \& Wesemael, F. 1995b, ApJ, 443, 764 
Bergeron, P., Wesemael, F., \& Beauchamp, A. 1995c, PASP, 107, 1047

Bergeron, P., Wesemael, F., Lamontagne, R., Fontaine, G., Saffer, R. A., \& Allard, N. F. 1995d, ApJ, 449, 258

Burleigh, M. R., Clarke, F. J., \& Hodgkin, S. T. 2002, MNRAS, 331, L41

Burleigh, M. R., Hogan, E., Dobbie, P. D., Napiwotzki, R., \& Maxted, P. F. L. 2006, MNRAS, 373, L55

Burleigh, M. R., et al. 2008, MNRAS, 386, L5

Burrows, A., Sudarsky, D., \& Lunine, J. I. 2003, ApJ, 596, 587

Chauvin, G., Lagrange, A. M., Dumas, C., Zuckerman, B., Mouillet, D., Song, I., Beuzit, J. L., \& Lowrance, P. 2005, A\&A, 438, L25

Cheselka, M., Holber, J., Watkins, R., Collins, J., \& Tweedy, R. W. 1993, AJ, 106,2365

Claver, C. F., Liebert, J., Bergeron, P., \& Koester, D. 2001, ApJ, 563, 987

Dahn, C. C., et al. 2002, AJ, 124, 1170

Debes, J. H., \& Sigurdsson, S. 2002, ApJ, 572, 556

Debes, J. H., Sigurdsson, S., \& Hansen, B. 2007, AJ, 134, 1662

Dobbie, P. D., Napiwotzki, R., Lodieu, N., Burleigh, M. R., Barstow, M. A., \& Jameson, R. F. 2006b, MNRAS, 373, L45

Dobbie, P. D., et al. 2006a, MNRAS, 369, 383

Dupuis, J., Vennes, S., \& Chayer, P. 2002, ApJ, 580, 1091

Eggen, O. J. 1984, AJ, 89, 830

Eggen, O. J., \& Greenstein, J. L. 1965, ApJ, 141, 83

Farihi, J. 2004, Ph.D. thesis, Univ. California, Los Angeles

Farihi, J., Becklin, E. E., \& Macintosh, B. A. 2004, ApJ, 608, L109

Farihi, J., Becklin, E. E., \& Zuckerman, B. 2005a, ApJS, 161, 394

Farihi, J., \& Christopher, M. 2004, AJ, 128, 1868

Farihi, J., Hoard, D. W., \& Wachter, S. 2006, ApJ, 646, 480

Farihi, J., Zuckerman, B., \& Becklin, E. E. 2005b, AJ, 130, 2237 2008, ApJ, 674, 431 (Paper I)

Fazio, G. G., et al. 2004, ApJS, 154, 10

Ferrario, L., Vennes, S., Wickramasinghe, D. T., Bailey, J. A., \& Christian, D. J. 1997, MNRAS, 292, 205

Ferrario, L., Wickramasinghe, D. T., Liebert, J., \& Williams, K. A. 2005, MNRAS, 361, 1131

Finley, D. S., Koester, D., \& Basri, G. 1997, ApJ, 488, 375

Friedrich, S., Zinnecker, H., Brandner, W., Correia, S., \& McCaughrean, M. 2005, in ASP Conf. Ser. 334, Proc. 14th European Workshop on White Dwarfs, ed. D. Koester \& S. Moehler (San Francisco: ASP), 431

Frink, S., Mitchell, D. S., Quirrenbach, A., Fischer, D. A., Marcy, G. W., \& Butler, R. P. 2002, ApJ, 576, 478

Glass, I. S. 1999, Handbook of Infrared Astronomy (Cambridge: Cambridge Univ. Press)

Han, Z., Podsiadlowski, P., Maxted, P. F. L., Marsh, T. R., \& Ivanova, N. 2002, MNRAS, 336, 449

Hansen, B. M. S., Kulkarni, S., \& Wiktorowicz, S. 2006, AJ, 131, 1106

Hatzes, A. P., Guenther, E. W., Endl, M., Cochran, W. D., Döllinger, M. P., \& Bedalov, A. 2005, A\&A, 437, 743

Hatzes, A. P., et al. 2006, A\&A, 457, 335

Hoard, D. W., Wachter, S., Sturch, L. K., Widhalm, A. M., Weiler, K. P., Pretorius, M. L., Wellhouse, J. W., \& Gibiansky, M. 2007, AJ, 134, 26

Hurley, J. R., Pols, O. R., \& Tout, C. A. 2000, MNRAS, 315, 543

Jeans, J. 1924, MNRAS, 85, 2

Jura, M. 2003, ApJ, 584, L91

Jura, M., Farihi, J., \& Zuckerman, B. 2007a, ApJ, 663, 1285

Jura, M., Farihi, J., Zuckerman, B., \& Becklin, E. E. 2007b, AJ, 133, 1927

Kalirai, J. S., Hansen, B. M. S., Kelson, D. D., Reitzel, D. B., Rich, R. M., \& Richer, H. B. 2008, ApJ, 676, 594

Kalirai, J. S., Richer, H. B., Reitzel, D., Hansen, B. M. S., Rich, R. M., Fahlman, G. G., Gibson, B. K., \& von Hippel, T. 2005, ApJ, 618, L123

Kilic, M., \& Redfield, S. 2007, ApJ, 660, 641

Kirkpatrick, J. D., Allard, F., Bida, T., Zuckerman, B., Becklin, E. E., Chabrier, G., \& Baraffe, I. 1999, ApJ, 519, 834

Koester, D., et al. 2001, A\&A, 378, 556

Liebert, J., Bergeron, P., \& Holberg, J. B. 2005a, ApJS, 156, 47

Liebert, J., Young, P. A., Arnett, D., Holberg, J. B., \& Williams, K. A. 2005b, ApJ, 630, L69
Liu, Y. J., et al. 2008, ApJ, 672, 553

Lovis, C., \& Mayor, M. 2007, A\&A, 472, 657

Makarov, V. V. 2004, ApJ, 600, L71

Marsh, M. C., et al. 1997, MNRAS, 286, 369

Maxted, P. F. L., Napiwotzki, R., Dobbie, P. D., \& Burleigh, M. R. 2006, Nature, 442, 543

McCook, G. P., \& Sion, E. M. 2006, Spectroscopically Identified White Dwarfs (Strasbourg: CDS)

Mermilliod, J. C. 1986, Catalog of Eggen's UBV Data (Strasbourg: CDS)

Mullally, F., Kilic, M., Reach, W. T., Kuchner, M. J., von Hippel, T., Burrows, A., \& Winget, D. E. 2007, ApJS, 171, 206

Mullally, F., Winget, D. E., Degennaro, S., Jeffery, E., Thompson, S. E., Chandler, D., \& Kepler, S. O. 2008, ApJ, 676, 573

Nakajima, T., Oppenheimer, B. R., Kulkarni, S. R., Golimowski, D. A., Matthews,

K., \& Durrance, S. T. 1995, Nature, 378, 463

Nelemans, G., \& Tauris, T. M. 1998, A\&A, 335, L85

Niedzielski, A., et al. 2007, ApJ, 669, 1354

Paczynski, B. 1976, in IAU Symp. 73, Structure and Evolution of Close Binary Systems, ed. P. Eggleton, S. Mitton, \& J. Whelan (Dordrecht: Reidel), 75

Patten, B. M., et al. 2006, ApJ, 651, 502

Perryman, M. A. C., et al. 1998, A\&A, 331, 81

Pinsonneault, M. H., Stauffer, J., Soderblom, D. R., King, J. R., \& Hanson, R. B. 1998, ApJ, 504, 170

Probst, R. 1983, ApJS, 53, 335

Rasio, F. A., Tout, C. A., Lubow, S. H., \& Livio, M. 1996, ApJ, 470, 1187

Reach, W. T., Kuchner, M. J., von Hippel, T., Burrows, A., Mullally, F., Kilic, M., \& Winget, D. E. 2005, ApJ, 635, L161

Reffert, S., Quirrenbach, A., Mitchell, D. S., Albrecht, S., Hekker, S., Fischer, D. A., Marcy, G. W., \& Butler, R. P. 2006, ApJ, 652, 661

Reid, I. N. 1993, MNRAS, 265, 785

Salim, S., \& Gould, A. 2003, ApJ, 582, 1011

Sato, B., et al. 2003, ApJ, 597, L157 2007, ApJ, 661, 527

Schreiber, M. R., \& Gänsicke, B. T. 2003, A\&A, 406, 305

Silvotti, R., et al. 2007, Nature, 449, 189

Smart, R. L., et al. 2003, A\&A, 404, 317

Soderblom, D. R., King, J. R., Hanson, R. B., Jones, B. F., Fischer, D. A., Stauffer, J. R., \& Pinsonneault, M. H. 1998, ApJ, 504, 192

Soker, N. 1998, AJ, 116, 1308

Song, I., Schneider, G., Zuckerman, B., Farihi, J., Becklin, E. E., Bessell, M. S., Lowrance, P., \& Macintosh, B. A. 2006, ApJ, 652, 724

Spitzer Science Center. 2006a, IRAC Data Handbook (ver. 3.0; Pasadena: SSC)

. 2006b, Spitzer Observer's Manual (ver. 7.1; Pasadena: SSC)

Stauffer, J. R., Schultz, G., \& Kirkpatrick, J. D. 1998, ApJ, 499, L199

Tremblay, P. E., \& Bergeron, P. 2007, ApJ, 657, 1013

Upgren, A. R., Weis, E. W., \& Hanson, R. B. 1985, AJ, 90, 2039

Vassiliadis, E., \& Wood, P. R. 1993, ApJ, 413, 641

Vennes, S. 1999, ApJ, 525, 995

Vennes, S., Ferrario, L., \& Wickramasinghe, D. T. 1999, MNRAS, 302, L49

Vennes, S., Schmidt, G. D., Ferrario, L., Christian, D. J., Wickramasinghe, D. T., \& Kawka, A. 2003, ApJ, 593, 1040

Vennes, S., Thej1l, P. A., Galvan, R. G., \& Dupuis, J. 1997, ApJ, 480, 714

Vennes, S., Thejll, P. A., Wickramasinghe, D. T., \& Bessell, M. S. 1996, ApJ, 467, 782

Villaver, E., \& Livio, M. 2007, ApJ, 661, 1192

Wachter, S., Hoard, D. W., Hansen, K. H., Wilcox, R. E., Taylor, H. M., \& Finkelstein, S. L. 2003, ApJ, 586, 1356

Weidemann, V. 1987, A\&A, 188, 74

. 1990, ARA\&A, 28, 103 2000, A\&A, 363, 647

Werner, M. W., et al. 2004, ApJS, 154, 1

Williams, K. A., Bolte, M., \& Koester, D. 2004, ApJ, 615, L49

Wolszczan, A., \& Frail, D. A. 1992, Nature, 355, 145

Zuckerman, B., \& Becklin, E. E. 1987, ApJ, 319, L99

Zuckerman, B., Koester, D., Reid, I. N., \& Hünsch, M. 2003, ApJ, 596, 477 